\title{
RANDOM MATRICES, GRAPHICAL ENUMERATION AND THE CONTINUUM LIMIT OF TODA LATTICES
}

\author{
N. M. ERCOLANI, K. D. T-R MCLAUGHLIN, AND V. U. PIERCE
}

\begin{abstract}
In this paper we derive a hierarchy of differential equations which uniquely determine the coefficients in the asymptotic expansion, for large $N$, of the logarithm of the partition function of $N \times$ $N$ Hermitian random matrices. These coefficients are generating functions for graphical enumeration on Riemann surfaces. The case that we particularly consider is for an underlying measure that differs from the Gaussian weight by a single monomial term of degree $2 \nu$. The coupling parameter for this term plays the role of the independent dynamical variable in the differential equations. From these equations one may deduce functional analytic characterizations of the coefficients in the asymptotic expansion. Moreover, this ode system can be solved recursively to explicitly construct these coefficients as functions of the coupling parameter. This analysis of the fine structure of the asymptotic coefficients can be extended to multiple coupling parameters and we present a limited illustration of this for the case of two parameters.
\end{abstract}

\section{Motivation And Background}

The study of the Unitary Ensembles (UE) of random matrices [20, begins with a family of probability measures on the space of $N \times N$ Hermitian matrices. The measures are of the form

$$
d \mu_{\mathbf{t}}=\frac{1}{\widetilde{Z}_{N}} \exp \left\{-N \operatorname{Tr}\left[V_{\mathbf{t}}(M)\right]\right\} d M,
$$

where the function $V_{\mathbf{t}}$ is a scalar function, referred to as the potential of the external field, or simply the "external field" for short. Typically it is taken to be a polynomial, and written as follows:

$$
V_{\mathbf{t}}=\frac{1}{2} \lambda^{2}+\sum_{j=1}^{v} t_{j} \lambda^{j}
$$

The partition function $\widetilde{Z}_{N}$, which appears as a normalization factor in the UE measures, plays a central role in random matrix theory and its applications. It can be reduced to an integration over the eigenvalues which takes a form proportional to the integral (1.1), below, for the particular case when $k=N$.

When all the coefficients $t_{k}$ in the external field are set equal to zero the associated ensemble, corresponding to $\mu_{0}$, is called the Gaussian Unitary Ensemble (GUE). Many simplifications occur in the Gaussian case (see [15] for explanations of any unfamiliar terms):

(1) The partition function, when all $t_{k}$ vanish, is a Gaussian integral, and can be evaluated exactly.

(2) The matrix moments, $\int\left\{\operatorname{Tr}\left(M^{j}\right)\right\}^{k} d \mu_{0}(M)$, can be evaluated, using Wick's lemma, in terms of pair correlations of the matrix entries of $M$ which are complex normal random variables.

(3) The terms in these Wick coupling expansions are, in the manner of Feynman diagrams, in 1-1 correspondence with certain labelled, oriented graphs.

These observations led to the conjecture [5, 13] that the logarithm of the partition function has an asymptotic expansion of the form:

$$
\log \left(\frac{\widetilde{Z}_{N}(\mathbf{t})}{\widetilde{Z}_{N}(\mathbf{0})}\right)=N^{2} e_{0}(\mathbf{t})+e_{1}(\mathbf{t})+\frac{1}{N^{2}} e_{2}(\mathbf{t})+\cdots
$$

K. D. T-R McLaughlin was supported in part by NSF grants DMS-0451495 and DMS-0200749, as well as a NATO Collaborative Linkage Grant "Orthogonal Polynomials: Theory, Applications, and Generalizations" Ref no. PST.CLG.979738. N. M. Ercolani and V. U. Pierce were supported in part by NSF grants DMS-0073087 and DMS-0412310. 
where the coefficients $e_{g}(\mathbf{t})$ should be locally analytic functions of $\mathbf{t}$. The Taylor coefficients of $e_{g}$ should enumerate topologically distinct labelled, connected oriented graphs that can be embedded into a Riemann surface of genus $g$ in such a way that the complement of the graph in the surface is a disjoint union of contractible cells. Such a construction is referred to as a $g$-map (see section 1.3 for a precise definition). The $e_{g}(t)$ are generating functions for counting the number of $g$-maps with given numbers of vertices of specified valence. This conjecture was proven in [15] for appropriate domains (see below). The present paper builds on these results to present a more detailed description of the coefficients $e_{g}(\mathbf{t})$ and related generating functions.

More precisely, our interest is to develop a systematic, rigorous description of the fine structure for the large $N$ asymptotics of the following family of integrals:

$$
\begin{aligned}
& Z_{N}^{(k)}\left(t_{1}, t_{2}, \ldots, t_{v}\right)= \\
& \int \ldots \int \exp \left\{-N^{2}\left[\frac{1}{N} \sum_{j=1}^{k} V\left(\lambda_{j} ; t_{1}, \ldots, t_{v}\right)-\frac{1}{N^{2}} \sum_{j \neq \ell} \log \left|\lambda_{j}-\lambda_{\ell}\right|\right]\right\} d^{k} \lambda, \\
& V\left(\lambda ; t_{1}, \ldots, t_{v}\right)=V_{\mathbf{t}}(\lambda)=V(\lambda)=\frac{1}{2} \lambda^{2}+\sum_{j=1}^{v} t_{j} \lambda^{j} .
\end{aligned}
$$

where the parameters $\left\{t_{1}, \ldots, t_{v}\right\}$ are assumed to be such that the integral converges. For example, one may suppose that $v$ is even, and $t_{v}>0$. We will sometimes refer to the following set of $\mathbf{t}=\left(t_{1}, \ldots, t_{v}\right)$ for which (1.1) converges. For any given $T>0$ and $\gamma>0$, define

$$
\mathbb{T}(T, \gamma)=\left\{\mathbf{t} \in \mathbb{R}^{v}:|\mathbf{t}| \leq T, t_{v}>\gamma \sum_{j=1}^{v-1}\left|t_{j}\right|\right\} .
$$

The parameter $k$ is an integer that grows with $N$ in such a way that $\lim _{N \rightarrow \infty} k / N=x$, where $x$ is a finite non-zero value whose role will be specified more precisely later.

In this paper we derive a hierarchy of differential equations which uniquely determine the coefficients in the asymptotic expansion of $\log Z_{N}^{(N)}$ for monic even coupling parameters; i.e., we present the $e_{g}\left(t_{2 \nu}\right)$, for arbitrary $\nu$, as solutions to a system of ordinary differential equations. From this one can deduce functional analytic characterizations of these coefficients. Moreover, this ode system can be solved recursively in $g$ to explicitly construct $e_{g}\left(t_{2 \nu}\right)$. We illustrate this process by constructing closed form expressions for $e_{g}\left(t_{2 \nu}\right)$, in which $\nu$ appears as a parameter, for low values of $g$. This analysis of the fine structure of the $e_{g}$ can be extended to multiple coupling parameters and we present a limited illustration of this for the case of two parameters: $e_{g}\left(t_{2 \nu_{1}}, t_{2 \nu_{2}}\right)$.

Remark In [2], the so-called "Loop Equation" method is used to obtain some information about the fine structure of the coefficients. This approach is based on a formal derivation of a hierarchy of equations for the Cauchy transform of the mean density of eigenvalues. This interesting approach is unsatisfactory in that it relies on several interchanges of singular limits whose justification requires analytical considerations beyond the existence of the complete asymptotic expansion of the partition function. These analytical considerations are the subject of a forthcoming paper by Ercolani and McLaughlin [16].

1.1. Leading Order Asymptotics. The leading order behavior of $Z_{N}^{(k)}\left(t_{1}, t_{2}, \ldots, t_{v}\right)$ is rather classical, and is known for a very wide class of external fields $V$ (see, for example, [19]). We will require the following result.

Theorem 1.1. There is $T_{0}>0$ and $\gamma_{0}>0$ so that for all $\mathbf{t} \in \mathbb{T}(\mathbf{T}, \gamma), x \in[1 / 2,1]$, and $k / N \rightarrow x$ as $k, N \rightarrow \infty$, the following holds true:

$$
\lim _{N \rightarrow \infty} \frac{1}{k^{2}} \log \left\{Z_{N}^{(k)}\left(t_{1}, t_{2}, \ldots, t_{v}\right)\right\}=-I\left(x, t_{1}, \ldots, t_{v}\right)
$$


where

$$
I\left(x, t_{1}, \ldots, t_{v}\right)=\quad \inf _{\text {Borel measures } \mu, \mu \geq 0, \int d \mu=1}\left[\frac{1}{x} \int V(\lambda) d \mu(\lambda)-\iint \log |\lambda-\mu| d \mu(\lambda) d \mu(\eta)\right]
$$

(2) There is a unique measure $\mu_{V}$ which achieves the infimum defined on the right hand side of (1.4). This measure is absolutely continuous with respect to Lebesgue measure, and

$$
\begin{aligned}
d \mu_{V} & =\psi d \lambda, \\
\psi(\lambda) & =\frac{1}{2 \pi} \chi_{(\alpha, \beta)}(\lambda) \sqrt{(\lambda-\alpha)(\beta-\lambda)} h(\lambda),
\end{aligned}
$$

where $h(\lambda)$ is a polynomial of degree $v-2$, which is strictly positive on the interval $[\alpha, \beta]$ (recall that the external field $V$ is a polynomial of degree $v$ ). The polynomial $h$ is defined by

$$
h(z)=\frac{1}{2 \pi i x} \oint \frac{V^{\prime}(s)}{\sqrt{(s-\alpha)} \sqrt{(s-\beta)}} \frac{d s}{s-z}
$$

where the integral is taken on a circle containing $(\alpha, \beta)$ and $z$ in the interior, oriented counterclockwise.

(3) There exists a constant $l$, depending on $V$ such that the following variational equations are satisfied by $\mu_{V}$ :

$$
\begin{aligned}
& \int 2 \log |\lambda-\eta|^{-1} d \mu_{V}(\eta)+x^{-1} V(\lambda) \geq l \text { for } \lambda \in \mathbf{R} \backslash \operatorname{supp}\left(\mu_{V}\right) \\
& \int 2 \log |\lambda-\eta|^{-1} d \mu_{V}(\eta)+x^{-1} V(\lambda)=l \text { for } \lambda \in \operatorname{supp}\left(\mu_{V}\right) .
\end{aligned}
$$

(4) The endpoints $\alpha$ and $\beta$ are determined by the equations

$$
\begin{aligned}
& \int_{\alpha}^{\beta} \frac{V^{\prime}(s)}{\sqrt{(s-\alpha)(\beta-s)}} d s=0 \\
& \int_{\alpha}^{\beta} \frac{s V^{\prime}(s)}{\sqrt{(s-\alpha)(\beta-s)}} d s=2 \pi x .
\end{aligned}
$$

(5) The endpoints $\alpha(x, \mathbf{t})$ and $\beta(x, \mathbf{t})$ are actually analytic functions of $\mathbf{t}$ and $x$, which possess smooth extensions to the closure of $\{x, \mathbf{t}: x \in[1 / 2,1], \mathbf{t} \in \mathbb{T}(T, \gamma)\}$. They also satisfy $-\alpha(1, \mathbf{0})=\beta(1, \mathbf{0})=2$. In addition, the coefficients of the polynomial $h(\lambda)$ are also analytic functions of $\mathbf{t}$ and $x$, with smooth extensions to the closure of $\{x, \mathbf{t}: x \in[1 / 2,1], \mathbf{t} \in \mathbb{T}(T, \gamma)\}$, with

$$
h(\lambda, x=1, \mathbf{t}=\mathbf{0})=1 \text {. }
$$

Remark The variational problem appearing in (1.4) is a fundamental component in the theory of random matrices, as well as integrable systems and approximation theory. It is well known, (see, for example, [24]), that under general assumptions on $V$, the infimum is achieved at a unique measure $\mu_{V}$, called the equilibrium measure. For external fields $V$ that are analytic in a neighborhood of the real axis, and with sufficient growth at $\infty$, the equilibrium measure is supported on finitely many intervals, with density that is analytic on the interior of each interval, behaving at worst like a square root at each endpoint, (see [10] and [1]).

Remark We call the reader's attention to the parameter, $x$, in the formulation of the variational problem. We will consider the variational problem for $x \in(0,1]$, and we are particularly interested in $x$ near 1 . This parameter represents the asymptotic ratio of $k$ to $N: x=\lim _{N \rightarrow \infty} k / N$.

Remark For a proof of (1.3), we refer the reader to [19, however this result is commonly known in the approximation theory literature.

Remark It will prove useful to adapt the following alternative presentation for the function $\psi$ :

$$
\psi(\lambda)=\frac{1}{2 \pi i} R_{+}(\lambda) h(\lambda), \lambda \in(\alpha, \beta)
$$


where the function $R(\lambda)$ is defined via $R(\lambda)^{2}=(\lambda-\alpha)(\lambda-\beta)$, with $R(\lambda)$ analytic in $\mathbb{C} \backslash[\alpha, \beta]$, and normalized so that $R(\lambda) \sim \lambda$ as $\lambda \rightarrow \infty$. The subscript \pm in $R_{ \pm}(\lambda)$ denotes the boundary value obtained from the upper (lower) half plane.

1.2. Complete Asymptotic Expansion. In [15] it was established that a complete large $N$ asymptotic expansion of 1.1 exists. In this paper we will use a straightforward generalization of this result:

Theorem 1.2. There is $T>0$ and $\gamma>0$ so that for $\mathbf{t} \in \mathbb{T}(\mathbf{T}, \gamma)$, and $x=k / N$ in a neighborhood of $x=1$, one has the $N \rightarrow \infty$ asymptotic expansion

$$
\log \left(\frac{Z_{N}^{(k)}(\mathbf{t})}{Z_{N}^{(k)}(\mathbf{0})}\right)=k^{2} e_{0}(x, \mathbf{t})+e_{1}(x, \mathbf{t})+\frac{1}{k^{2}} e_{2}(x, \mathbf{t})+\cdots .
$$

The meaning of this expansion is: if you keep terms up to order $k^{-2 h}$, the error term is bounded by $C k^{-2 h-2}$, where the constant $C$ is independent of $x$ and $\mathbf{t}$ for all $\mathbf{t} \in \mathbb{T}(\mathbf{T}, \gamma)$ and for all $x$ in the neighborhood of 1 . For each $j$, the function $e_{j}(x, \mathbf{t})$ is an analytic function of the (complex) vector $(x, \mathbf{t})$, in a neighborhood of $(1, \mathbf{0})$. Moreover, the asymptotic expansion of derivatives of $\log \left(Z_{N}^{(k)}\right)$ may be calculated via term-by-term differentiation of the above series.

Remark In [15], this result was established in the case where $x=1$, and under the assumption that $\mathbf{t} \in$ $\mathbb{T}(T, \gamma)$, for $T$ small enough, and $\gamma$ large enough, so that Theorem 1.2 holds true. Under these assumptions, Theorem 1.3 (below) was established. However, as observed in [15] (Remark 2.1, page 2), the domain so defined is by no means the largest domain where the asymptotic expansion can be rigorously established. All that is required is the existence of a path through the space of parameters (values of $x$ and $\mathbf{t}$ ) connecting $(x, \mathbf{t})$ to $(1, \mathbf{0})$ in such a way that all along the path, the associated equilibrium measure is supported on a single interval, with strict variational inequality on the support, strict positivity on the interval of support, and vanishing like a square root at both endpoints of the support. The collection of all such values of $(x, \mathbf{t})$ defines a suitable candidate for a maximal domain, and the proof contained in [15] can easily be extended to show that the asymptotic expansion of the partition function holds on the interior of such a domain. In particular, the above Theorem may be easily deduced along these lines.

Remark Recently, Bleher and Its 8 , have carried out a similar asymptotic expansion of the partition function for a 1-parameter family of external fields. A very interesting aspect of their work is that they establish the nature of the asymptotic expansion of the partition through a critical phase transition.

1.3. Graphical Enumeration and the Partition Function Expansion. Our goal in the work we present here is to establish analytical characterizations of the coefficients $e_{g}$ and, when possible, to derive explicit expressions for these coefficients. This is what we mean by the fine structure of the expansion.

In addition to providing the first proof of the asymptotic expansion described in Theorem [1.2] [15] also provides a very detailed explanation of the connection between the asymptotic expansion and enumerative geometry, originally investigated by physicists in the 70s and 80s (see, for example, [5], 13], and references contained therein). Equipped with the existence of the asymptotic expansion (and the subsequent result that it may be differentiated term by term), one shows that there is a geometric characterization of each $e_{g}$ as a generating function for enumerating topologically distinct embeddings of graphs into Riemann surfaces of genus $g$.

A map $D$ on a compact, oriented connected surface $\mathrm{X}$ is a pair $D=(K(D),[\imath])$ where

(1) $K(D)$ is a connected 1-complex;

(2) $[\imath]$ is an isotopical class of inclusions $\imath: K(D) \rightarrow X$;

(3) the complement of $K(D)$ in $X$ is a disjoint union of open cells (faces);

(4) the complement of $K_{0}(D)$ (vertices) in $K(D)$ is a disjoint union of open segments (edges).

The $e_{g}$ enumerate labelled maps. To be precise we introduce the notion of a $g$-map which is a map in which the surface $\mathrm{X}$ is the closed, oriented Riemann surface of genus $\mathrm{g}$ and which in addition carries a labelling (ordering) of the vertices. 
Theorem 1.3. 15] The coefficients in the asymptotic expansion [1.7) satisfy the following relations. Let $g$ be a nonnegative integer. Then

$$
e_{g}\left(t_{1} \ldots t_{v}\right)=\sum_{n_{j} \geq 1} \frac{1}{n_{1} ! \ldots n_{v} !}\left(-t_{1}\right)^{n_{1}} \ldots\left(-t_{v}\right)^{n_{v}} \kappa_{g}\left(n_{1}, \ldots, n_{v}\right)
$$

in which each of the coefficients $\kappa_{g}\left(n_{1}, \ldots, n_{v}\right)$ is the number of g-maps with $n_{j} j$-valent vertices for $j=$ $1, \ldots, v$.

1.4. Outline. The organization of this paper is as follows: In section 2 we present the new results concerning the fine structure of the $e_{g}$ and related generating functions that will be proven and further explained in the remainder of the paper.

Section 3 is concerned with the leading order term, $e_{0}$. The results here are fundamental for the characterization of all the higher order terms. We derive closed form expressions for $e_{0}$ as a function of each of the valence coupling parameters $t_{2 \nu}$. We also relate these evaluations directly and explicitly to the enumeration of planar graphs.

In section 4 a continuum limit of the Toda Lattice hierarchy is rigorously derived in which the hierarchy of Toda times corresponds to the valence coupling parameters $t_{2 \nu}$. This continuum limit is then used to derive another hierarchy of differential equations whose solutions are the $e_{g}$.

Finally in section 5 we show how the differential equations derived in the previous section are used to inductively generate explicit expressions for the $e_{g}$. From this we characterize the function-theoretic structure of the $e_{g}$ as well as present explicit formulae for the $e_{g}$ for low values of $g$. We also show how our results may be extended to the case of multiple times.

\section{Results}

For $e_{0}$ we have explicit formulas for monic even times

Theorem 2.1. For potentials $V$ of the form $V=\frac{1}{2} \lambda^{2}+t_{2 \nu} \lambda^{2 \nu}$, the asymptotic expansion (1.7) holds true for all $t_{2 \nu} \geq 0$, and in addition, we have the explicit formula

$$
e_{0}=\eta(z-1)(z-r)+\frac{1}{2} \log (z)
$$

where

$$
\begin{aligned}
& \eta=\frac{(\nu-1)^{2}}{4 \nu(\nu+1)}, \\
& r=\frac{3(\nu+1)}{\nu-1}, \\
& z=\frac{\beta^{2}}{4} .
\end{aligned}
$$

Here $4 z$ can be interpreted as the global analytic continuation of $\beta^{2}$ which determines the support $(-\beta, \beta)$ of the equilibrium measure. The variable $z$ is locally an analytic function of $t_{2 \nu}$, which satisfies the algebraic relation

$$
1=z+2 \nu\left(\begin{array}{c}
2 \nu-1 \\
\nu-1
\end{array}\right) x^{\nu-1} t_{2 \nu} z^{\nu}
$$

The singularities of $e_{0}$ occur at $z=0$ and $z=\infty$. The time derivative

$$
\frac{\partial e_{0}}{\partial t_{2 \nu}}=\left(\begin{array}{c}
2 \nu-1 \\
\nu-1
\end{array}\right) z^{\nu}((\nu-1) z-(\nu+1))
$$

is polynomial in $z$. 
One also has a local analytical representation (here the index $n_{2 \nu}$ is replaced by $n$, so that $\kappa_{0}\left(0, \ldots, 0, n_{2 \nu}\right)$ becomes $\left.\kappa_{0}(n)\right)$,

$$
\begin{aligned}
e_{0}\left(t_{2 \nu}\right) & =\sum_{j=1}^{\infty} \kappa_{0}(n) \frac{\left(-t_{2 \nu}\right)^{n}}{n !}, \\
\kappa_{0}(n) & =\left(c_{\nu}\right)^{n} \frac{(\nu n-1) !}{((\nu-1) n+2) !} \\
c_{\nu} & =2 \nu\left(\begin{array}{c}
2 \nu-1 \\
\nu-1
\end{array}\right),
\end{aligned}
$$

where $\kappa_{0}(n)=\kappa_{0}\left(n_{2 \nu}\right)$ is the generating function for $2 \nu$-valent 0 -maps.

To get a handle on how the higher coefficients $e_{g}$ depend on the parameters $t=t_{2 \nu}$ we exploit a remarkable relation between the partition function $Z_{N}^{(N)}(\mathbf{t})$ and the solutions to the hierarchy of completely integrable semi-infinite Toda lattice equations. These differential equations may be succinctly expressed through the semi-infinite tri-diagonal matrix

$$
\mathcal{L}=\left(\begin{array}{ccccc}
0 & 1 & 0 & 0 & \cdots \\
b_{0}^{2} & 0 & 1 & 0 & \cdots \\
0 & b_{1}^{2} & \ddots & \ddots & \ddots \\
0 & 0 & \ddots & 0 & 1 \\
\vdots & \vdots & \ddots & b_{n}^{2} & \ddots
\end{array}\right)
$$

The Toda Lattice system at level $2 \nu$ can then be defined as

$$
\begin{aligned}
\frac{1}{2} \frac{d b_{k}^{2}}{d \xi} & =\left(\mathcal{L}^{2 \nu}\right)_{k+2, k}-\left(\mathcal{L}^{2 \nu}\right)_{k+1, k-1} \\
\left(\mathcal{L}^{2 \nu}\right)_{k+1, k-1} & =\sum_{i_{1}, i_{2}, \ldots, i_{2 \nu+1} ;\left|i_{j+1}-i_{j}\right|=1 ; i_{1}=k+1, i_{2 \nu+1}=k-1} \mathcal{L}_{k+1, i_{2}} \mathcal{L}_{i_{2}, i_{3}} \ldots \mathcal{L}_{i_{2 \nu}, k-1}
\end{aligned}
$$

The sum here is indexed by walks of length $2 \nu$ along the $1 \mathrm{D}$ integer lattice from $k+1$ to $k-1$. The solution of this system may be expressed directly in terms of the partition function $Z_{k}\left(t_{1}, t\right)=Z_{k}^{(k)}$ associated to the potential $V=\frac{1}{2} \lambda^{2}+t_{1} \lambda+t \lambda^{2 \nu}$ :

$$
b_{k}^{2}(\xi)=k\left(\frac{1}{2 k^{2}}\right) \frac{d^{2}}{d t_{1}^{2}} \log Z_{k}\left(t_{1}, s\right)_{t_{1}=-k^{-1 / 2}} \xi_{1}=0, s=2 \xi k^{\nu-1} .
$$

As a dynamical system, one is really considering an initial value problem, with

$$
b_{k}(0)^{2}=k \text {. }
$$

We can now state our next main result which characterizes the continuum limit of the Toda lattice hierarchy.

Theorem 2.2. For all $t \geq 0, b_{k}^{2}$ has a valid asymptotic expansion of the form

$$
b_{k}^{2} \simeq k\left(z_{0}(s)+\frac{1}{k^{2}} z_{1}(s)+\frac{1}{k^{4}} z_{2}(s)+\cdots\right)
$$

where $s=-2 k^{\nu-1} t$. The terms of this expansion are determined by the following partial differential scheme:

$$
f_{s}=c_{\nu} f^{\nu} f_{w}+\frac{1}{k^{2}} F_{1}^{(\nu)}\left(f, f_{w}, f_{w w}, f_{w w w}\right)+\cdots+\frac{1}{k^{2 g}} F_{g}^{(\nu)}\left(f, f_{w}, f_{w^{(2)}}, \cdots, f_{w^{(2 g+1)}}\right)+\left.\cdots\right|_{\text {evaluated at } w=1} ;
$$

where

$$
\begin{aligned}
f(s, w) & =f_{0}(s, w)+\frac{1}{k^{2}} f_{1}(s, w)+\cdots+\frac{1}{k^{2 g}} f_{g}(s, w)+\cdots, \text { and } \\
f(s, 1) & =z_{0}(s)+\frac{1}{k^{2}} z_{1}(s)+\frac{1}{k^{4}} z_{2}(s)+\cdots \\
f_{g}(s, w) & =w^{1-2 g} z_{g}\left(w^{\nu-1} s\right) .
\end{aligned}
$$


Note that $b_{k}^{2}$ and $k f(s, 1)$ possess the same asymptotic expansion.

The forcing term $\left.F_{j}^{(\nu)}(\cdots)\right|_{w=1}$ is a homogeneous multi-nomial of degree $\nu+1$ in the $f_{w^{(r)}}$ which does not contain any instances of $z_{\alpha}$ for $\alpha \geq j$.

These forcing terms have the following form:

$$
F_{g}^{(\nu)}=\sum_{V:|V|=2 g+1 \ni \rho(V) \leq \nu+1} d_{V}^{(\nu, g)} f^{\nu-\rho(V)+1} \prod_{j=1}^{2 g+1}\left(\frac{f_{w^{(j)}}}{j !}\right)^{r_{j}(V)}
$$

where $V=\bigcup_{m=1}^{\rho(V)} V_{m}$ is a partition of $2 g+1 ; r_{j}$ is the number of times a "part", $V_{m}$, of cardinality $\left|V_{m}\right|=j$ appears in the partition; $\rho=\rho(V)=\sum r_{j}(V)$; and

$$
\begin{aligned}
d_{V}^{(\nu, g)} & =\frac{1}{\prod_{j=1}^{2 g+1} r_{j} !} \sum_{1 \leq i_{1}<\cdots<i_{\rho(V)} \leq 2 \nu}\left(\text { coeff of } x^{\nu-\rho(V)+1} \text { in } P(x)-\text { coeff of } x^{\nu-\rho(V)+1} \text { in } Q(x)\right) ; \text { where }, \\
P(x) & =\sum_{\sigma \in \mathcal{S}} \prod_{m=1}^{\rho(V)}\left(i_{m}-2 \sum_{s=1}^{m}\left(1+x_{s} \frac{\partial}{\partial x_{s}}\right)+2\right)^{\left|V_{\sigma(m)}\right|} \\
& \left.\cdot\left(1+x_{1}\right)^{i_{1}-1} \cdots\left(1+x_{s}\right)^{i_{s}-i_{s-1}-1} \cdots\left(1+x_{\rho}\right)^{i_{\rho}-i_{\rho-1}-1}\left(1+x_{\rho+1}\right)^{2 \nu-i_{\rho}}\right|_{x_{\mu}=x}, \\
Q(x)= & \sum_{\sigma \in \mathcal{S}} \prod_{m=1}^{\rho(V)}\left(i_{m}-2 \sum_{s=1}^{m}\left(1+x_{s} \frac{\partial}{\partial x_{s}}\right)+1\right)^{\left|V_{\sigma(m)}\right|} \\
& \left.\cdot\left(1+x_{1}\right)^{i_{1}-1} \cdots\left(1+x_{s}\right)^{i_{s}-i_{s-1}-1} \cdots\left(1+x_{\rho+1}\right)^{2 \nu-i_{\rho}}\right|_{x_{\mu}=x} .
\end{aligned}
$$

Remark We refer to the above as a partial differential scheme because it signifies not an equation to be solved but rather a prescription for generating a hierarchy of ordinary differential equations for the $z_{g}$. The ode hierarchy is constructed from the scheme as follows. The ode at level $g$ is obtained by replacing the expansion $f$ and its $w$-derivatives, $f_{w}, f_{w w}$, etc., in the pde scheme by their order $g$ truncations. Then, equating the coefficients of all terms of order $k^{-2 g}$ in this truncated scheme and setting $w=1$ yields a $k$-independent ode in $s$ which is the $g^{t h}$ equation of the continuum-Toda hierarchy. This is an ode for $z_{g}(s)$ in terms of $z_{j}(s)$ for $j \leq g$.

The first equation in the hierarchy (i.e., the one coming from the $k^{0}$-coefficients of the above scheme) is a nonlinear ODE for $z_{0}$ :

$$
z_{0}^{\prime}(s)=c_{\nu} z_{0}(s)^{\nu}\left(z_{0}(s)+(\nu-1) s z_{0}^{\prime}(s)\right),
$$

with the initial condition $z_{0}(0)=1$. This ODE is solved implicitly by a solution to the algebraic equation

$$
1=z_{0}(s)-c_{\nu} s z_{0}(s)^{\nu} .
$$

As indicated in Theorem 1.1 (5), this relation can also be derived directly from the characterization of the equilibrium measure. Relation (2.6) allows us to write $s$ as well as derivatives of $z_{0}$ as rational functions of $z_{0}$. This will be exploited to arrive at the explicit representations of the $z_{g}$ given below.

The $k^{-2 g}$ equation in the hierarchy is linear in $z_{g}$, and can be written as:

$$
z_{g}^{\prime}(s)=c_{\nu}\left(f_{0}^{\nu} f_{g_{w}}+\nu f_{0}^{\nu-1} f_{g} f_{0 w}\right)_{w=1}+\text { Forcing }\left._{g}\right|_{w=1}
$$

where

(2.8) Forcing $_{g}=\left(\begin{array}{c}\frac{c_{\nu}}{\nu+1} \frac{\partial}{\partial w} \sum_{\substack{0 \leq i_{j}<g \\ i_{1}+\cdots+i_{\nu+1}=g}} f_{i_{1}} \cdots f_{i_{\nu+1}}\end{array}\right)+F_{1}^{(\nu)}[2 g-2]+F_{2}^{(\nu)}[2 g-4]+\cdots+F_{g}^{(\nu)}[0]$,

and $F_{\ell}^{(\nu)}[2 r]$ denotes the coefficient of $k^{-2 r}$ in $F_{\ell}^{(\nu)}$. We note that the terms in Forcing $\left.\left.\right|_{g}\right|_{w=1}$ depend only on $z_{j}, j<g$ and their derivatives. 
Remark Amongst the results on the above singular limit of the Toda lattice in the literature, we remark that the recent work of Bloch, Uribe, and Golse [6] is related, in the sense that in their work, through the use of the theory of Toeplitz operators, the authors establish the existence of an asymptotic expansion for a continuum limit of a finite dimensional Toda lattice.

For the case of planar maps $(g=0)$ such generating functions have received significant attention recently [7. 9]. Our explicit calculations presented later can provide a basis for extending these studies.

Before proceeding to the statement of our next result we need to introduce a scale of function classes that will enable us to describe the functional nature of the coefficients $z_{g}$ that we have just introduced as well as that of the generating functions $e_{g}$. We will refer to the classes as iterated integrals of rational functions or iir for short. These classes are defined inductively in terms of the variable $z=z_{0}$ regarded as an independent variable as follows. To begin with, the class contains rational functions of $z$. One then adds integrals of these rational functions with respect to $d z$. Next one considers the vector space of polynomials in products of these integrals over the field of rational functions in $z$ and augments the space by integrals, with respect to $d z$ of these functions. Then take the vector space of polynomials in these latter integrals and add integrals of these. One continues this iterative process up to any given finite stage. These are the classes of functions we refer to as iir. In our case the rational functions at any stage will be restricted to the sub-ring of functions whose poles are located at either $z=0$ or $z=\nu /(\nu-1)$ for a fixed value of $\nu$. These classes of functions will certainly include the class generated by polylogarithms [17] but may be larger.

Theorem 2.3. (1) The coefficient $z_{g}$ is of class iir in $z_{0}$ with singularities only possible at $z_{0}=0$ and $z_{0}=\nu /(\nu-1)$.

(2) The coefficient $z_{g}$ is more explicitly presented as a function of $z_{0}$ through the following integral solution of Equation 2.7):

$$
z_{g}(s)=\frac{z_{0}(s)^{2(1-g)}}{\nu-(\nu-1) z_{0}(s)} \int_{1}^{z_{0}(s)} \frac{(\nu-(\nu-1) y)}{c_{\nu} y^{\nu+3-2 g}} \operatorname{Forcing}_{g}(y) d y
$$

(3) In the above equation, Forcing ${ }_{g}$, formerly a function of a great many arguments, is in fact a function of $z_{0}(s)$ alone, which will henceforth be denoted as Forcing ${ }_{g}\left(z_{0}\right)$.

We also derive a hierarchy of differential equations for the $e_{g}\left(t_{2 \nu}\right)$ with data given in terms of the $z_{j}^{\prime} s$.

Theorem 2.4. The g'th equation in the hierarchy of equations governing $e_{g}\left(t_{2 \nu}\right)$ is

$$
\begin{aligned}
\left.\frac{\partial^{2}}{\partial w^{2}}\left[w^{2-2 g} e_{g}\left(-w^{\nu-1} s\right)\right]\right|_{w=1} & =-\left.\sum_{n=1}^{g} \frac{2}{(2 n+2) !} \frac{\partial^{(2 n+2)}}{\partial w^{(2 n+2)}}\left[w^{2-2(g-n)} e_{g-n}\left(-w^{\nu-1} s\right)\right]\right|_{w=1} \\
& + \text { the } k^{-2 g} \text { term of } \log \left(\sum_{n=0}^{\infty} \frac{1}{k^{2 n}} z_{n}(s)\right) .
\end{aligned}
$$

Equation (2.9) determines $e_{g}(-s)$ from a second order differential equation for $e_{g}$ with forcing terms depending on $e_{n}, n<g, z_{n}, n \leq g$, and their derivatives.

Remark Observe that the RHS of (2.9) is a function of $z_{0}(s)$ which will henceforth be denoted by drivers $g_{g}\left(z_{0}\right)$.

Theorem 2.5. (1) The coefficient $e_{g}(-s)$ is of class iir in $z_{0}$ with singularities restricted to $z_{0}=0$ and $z_{0}=\nu /(\nu-1)$. 
(2) The solution of (2.9) may be represented as

$$
\begin{aligned}
e_{g}(-s) & =-\frac{1}{(2-2 g)(1-2 g)} \operatorname{drivers}_{g}\left(z_{0}(s)\right) \\
& -\frac{1}{2-2 g}\left(\frac{c_{\nu} z_{0}(s)^{\nu}}{z_{0}(s)-1}\right)^{(2-2 g) /(\nu-1)} \int_{1}^{z_{0}(s)}\left(\frac{y-1}{c_{\nu} y^{\nu}}\right)^{(2-2 g) /(\nu-1)}\left(\operatorname{drivers}_{g}(y)\right)^{\bullet} d y \\
& +\frac{1}{(1-2 g)}\left(\frac{c_{\nu} z_{0}(s)^{\nu}}{z_{0}(s)-1}\right)^{(1-2 g) /(\nu-1)} \int_{1}^{z_{0}(s)}\left(\frac{y-1}{c_{\nu} y^{\nu}}\right)^{(1-2 g) /(\nu-1)}\left(\operatorname{drivers}_{g}(y)\right)^{\bullet} d y \\
& +K_{1} s^{(2 g-2) /(\nu-1)}+K_{2} s^{(2 g-1) /(\nu-1)}
\end{aligned}
$$

when $g \neq 1$, where $K_{1}$ and $K_{2}$ are constants of integration either determined by the requirement that $e_{g}$ be a locally analytic function of $s$ or by the evaluation of $e_{g}$ for low values of $\nu$ through its combinatorial characterization; and, when $g=1$,

$$
\begin{aligned}
e_{1}(-s)=\frac{1}{(\nu-1)} & {\left[\left(\frac{z_{0}(s)-1}{c_{\nu} z_{0}(s)^{\nu}}\right)^{1 /(\nu-1)} \int_{1}^{z_{0}(s)}\left(\frac{c_{\nu} y^{\nu}}{y-1}\right)^{\nu /(\nu-1)} \frac{(\nu-(\nu-1) y)}{c_{\nu} y^{\nu+1}} \operatorname{drivers}_{1}(y) d y\right.} \\
& \left.-\int_{1}^{z_{0}(s)} \frac{(\nu-(\nu-1) y)}{y(y-1)} \operatorname{drivers}_{1}(y) d y\right] \\
= & -\frac{1}{12} \log \left(\nu-(\nu-1) z_{0}(s)\right)
\end{aligned}
$$

where we have chosen the principal branch of the logarithm. By $\left(\operatorname{drivers}_{g}(y)\right)^{\bullet}$ we mean the derivative of $\operatorname{drivers}_{g}(y)$ with respect to $y$.

\section{LEADING ORDER}

We will show that the leading order coefficient, $e_{0}(x, t)$, of the asymptotic partition function, $k^{-2} \log \left(Z_{N}^{(k)}(t) / Z_{N}^{(k)}(0)\right)$ is found in terms of the equilibrium measure $\mu=\mu_{V_{t} / x}$. Define $\psi(\lambda)$ by

$$
d \mu=\psi(\lambda) d \lambda \text {. }
$$

We denote the leading order behavior

$$
E_{t}=\lim _{k \rightarrow \infty}-\frac{1}{k^{2}} \log \left(Z_{N}^{(k)}(t)\right)=I(x, t),
$$

and

$$
\lim _{k \rightarrow \infty}-\frac{1}{k^{2}} \log \left(\frac{Z_{N}^{(k)}(t)}{Z_{N}^{(k)}(0)}\right)=E_{t}-E_{0}
$$

More explicitly, by (1.4)

$$
\begin{aligned}
E_{t} & =\int \frac{V_{t}(\lambda)}{x} d \mu(\lambda)+\iint \log |\lambda-\eta|^{-1} d \mu(\lambda) d \mu(\eta) \\
& =\left(\frac{V_{t}(\lambda)}{x}, \psi(\lambda)\right)-(\mathcal{L} \psi, \psi),
\end{aligned}
$$

where

$$
(\mathcal{L} f)(\lambda)=\int \log |\lambda-\eta| f(\eta) d \eta
$$

is the logarithmic potential of the measure $f(\eta) d \eta$, and where the inner product $(\cdot, \cdot)$ is defined by

$$
(f, g)=\int f(\lambda) g(\lambda) d \lambda
$$

Using (3.1) and (3.2) together with (1.7), we find that

$$
e_{0}(t, x)=-\frac{1}{x}\left(\frac{1}{2} \lambda^{2}+t \lambda^{2 \nu}, \psi(\lambda)\right)+(\mathcal{L} \psi, \psi)+\frac{1}{x}\left(\frac{1}{2} \lambda^{2}, \psi_{0}(\lambda)\right)-\left(\mathcal{L} \psi_{0}, \psi_{0}\right) .
$$


We recall here the parameter $l$ introduced in (1.5):

$$
\begin{aligned}
l & =\int 2 \log |\lambda-s|^{-1} \psi(s) d s+\frac{V(\lambda)}{x} \\
& =-2(\mathcal{L} \psi, \psi)+\frac{V(\lambda)}{x} .
\end{aligned}
$$

Using this we have the following reduced formula for $e_{0}$ :

$$
e_{0}=-\frac{(V, \psi)}{2 x}+\frac{(-l)}{2}+\frac{1}{4 x}\left(\lambda^{2}, \psi_{0}\right)-\frac{\left(-l_{0}\right)}{2} .
$$

This formula shows that there are two fundamental quantities that need to be calculated in order to evaluate $e_{0}$. These are the moment $(V, \psi)$ and the lagrange multiplier $l$ associated to the constraint that the measure $\mu$ should have total mass $=1$. The other quantities appearing in (3.4), $\left(\lambda^{2}, \psi_{0}\right)$ and $l_{0}$ are evaluated by specializing the fundamental quantities at $t=0$. Evaluating the fundamental quantities will require an explicit asymptotic expansion, for large $\lambda$, of the equilibrium measure $\psi(\lambda) d \lambda$. We develop this in the next section.

3.1. Explicit asymptotic expansion of the equilibrium measure. The equilibrium measure $\mu$ is of the form

$$
\psi(\lambda)=\frac{1}{2 \pi i} h(\lambda) \sqrt{\lambda^{2}-\beta^{2}} \chi_{[-\beta, \beta]}(\lambda),
$$

where $h$ is a polynomial determined by

$$
h(\lambda)=\frac{1}{2 \pi i} \oint \frac{V_{t}^{\prime}(s)}{x \sqrt{s^{2}-\beta^{2}}(s-\lambda)} d s,
$$

the integral in (3.6) is taken along a simple closed counterclockwise contour large enough to contain the interval $[-\beta, \beta]$ and the point $\lambda$.

We will now evaluate the polynomial $h$ by expanding the integrand of (3.6) for large $\lambda$ and calculating the loop integral on this expansion.

Define the sequence $\left\{v_{j}\right\}_{j=0}^{\infty}$ by

$$
\frac{\sqrt{\lambda^{2}-\beta^{2}}}{\lambda}=1-\sum_{i=0}^{\infty} v_{i} \frac{1}{\lambda^{2 i+2}} ;
$$

whose Taylor coefficients can be computed to be

$$
v_{i}=\frac{1}{4^{i}}\left(\begin{array}{c}
2 i-1 \\
i-1
\end{array}\right) \frac{\beta^{2 i+2}}{i+1},
$$

with $v_{0}$ defined to be $\beta^{2} / 2$.

Expand the polynomial $h$, given by (3.6), in terms of its coefficients $h_{j}$ :

$$
h(\lambda)=\frac{1}{x}\left(1+\sum_{j=0}^{\nu-1} h_{j} \lambda^{2 j}\right) .
$$

Next note that

$$
\frac{\lambda}{\sqrt{\lambda^{2}-\beta^{2}}}=\sum_{i=0}^{\infty} \frac{2(i+1) v_{i}}{\beta^{2}} \frac{1}{\lambda^{2 i}} .
$$

A direct computation of (3.6) gives

$$
h_{j}=4 \nu(\nu-j) t \frac{v_{\nu-1-j}}{\beta^{2}} .
$$

The constraint that the total mass of $\mu$ should be 1 ,

$$
1=\int_{-\beta}^{\beta} \psi(\lambda) d \lambda=\frac{1}{2} \oint \psi(\lambda) d \lambda,
$$


implicitly determines $t$ as a function of $\beta^{2}$. Here, the integral is over a contour containing the interval $[-\beta, \beta]$.

We compute the loop integral in (3.12) over a large contour and find that the constraint can be expressed as a relation between the coefficients $\left\{h_{j}\right\}$ and $\left\{v_{j}\right\}$ :

$$
2=\frac{v_{0}}{x}+\sum_{j=0}^{\nu-1} \frac{h_{j} v_{j}}{x} .
$$

The relation (3.13) simplifies to the expression (using identities (3.8) and 3.11)

$$
2 x=\frac{\beta^{2}}{2}+\frac{\nu}{4^{\nu-1}}\left(\begin{array}{c}
2 \nu-1 \\
\nu-1
\end{array}\right) t \beta^{2 \nu} .
$$

If we set $z=\beta^{2} /(4 x)$ becomes

$$
1=z+2 \nu\left(\begin{array}{c}
2 \nu-1 \\
\nu-1
\end{array}\right) x^{\nu-1} t z^{\nu}
$$

When $\nu=2$,

$$
h(\lambda)=\frac{1}{x}\left(1+2 t \beta^{2}+4 t \lambda^{2}\right)
$$

with the constraint

$$
1=z+12 x t z^{2}
$$

3.2. Explicit calculation of $l$. We will first derive an analytic expression for $l$ in terms of $\beta^{2}$. To this end we study the logarithmic potential of $\mu$ :

$$
g(\lambda)=\int_{-\beta}^{\beta} \log (\lambda-s) \psi(s) d s .
$$

This function is analytic in $\mathbb{C} \backslash(-\infty, \beta]$. For $\lambda \in(-\infty, \beta]$ we define two functions $g_{+}$and $g_{-}$by

$$
g_{ \pm}(\lambda)=\lim _{\epsilon \rightarrow 0} g(\lambda \pm i \epsilon) .
$$

Choosing the appropriate branch of the logarithm we find that these functions are expressible as

$$
g_{ \pm}(\lambda)=\int \log |\lambda-s| \psi(s) d s \pm i \pi \int_{\lambda}^{\beta} \psi(s) d s
$$

A calculation using (3.19) and (1.5) shows that

$$
g_{+}(\lambda)+g_{-}(\lambda)-\frac{V(\lambda)}{x}+l=2 \int \log |\lambda-s| \psi(s) d s-\frac{V(\lambda)}{x}+l=0,
$$

and

$$
g_{+}(\lambda)-g_{-}(\lambda)=-2 i \pi \int_{\beta}^{\lambda} \psi(s) d s
$$

Equation (3.21) implies that

$$
g_{+}(\lambda)=g_{-}(\lambda)-2 i \pi \int_{\beta}^{\lambda} \psi(s) d s
$$

therefore, if $\lambda$ is in $\mathbb{C} /(-\infty, \beta]$, then equation (3.20) becomes

$$
2 g(\lambda)-2 i \pi \int_{\beta}^{\lambda} \psi(s) d s-\frac{V(\lambda)}{x}+l=0,
$$

and

$$
-l=2 g(\lambda)-\frac{V(\lambda)}{x}+\int_{\beta}^{\lambda} h(s) \sqrt{s^{2}-\beta^{2}} d s .
$$


This is the basic expression we shall use to calculate $l$. Since $l$ is a constant it can be evaluated for any choice of $\lambda$. We will evaluate it by studying the limit of (3.22) as $\lambda \rightarrow \infty$. First observe that in this limit

$$
g(\lambda)=\log (\lambda)+\mathcal{O}\left(\frac{1}{\lambda}\right)
$$

Thus the principal issue is to develop an asymptotic expansion of the indefinite integral

$$
\int_{\beta}^{\lambda} h(s) \sqrt{s^{2}-\beta^{2}} d s .
$$

The details of the derivation are deferred to Appendix $\mathrm{A}$ the result is the following:

$$
\begin{aligned}
\int_{\beta}^{\lambda} h(s) \sqrt{s^{2}-\beta^{2}}= & \frac{1}{x} W(\lambda)\left(\lambda^{2}-\beta^{2}\right) \frac{\sqrt{\lambda^{2}-\beta^{2}}}{\lambda}+\frac{2}{\beta^{2}} \lambda^{2} \frac{\sqrt{\lambda^{2}-\beta^{2}}}{\lambda} \\
& -2 \log \left(\frac{\lambda}{\beta}+\frac{\sqrt{\lambda^{2}-\beta^{2}}}{\beta}\right),
\end{aligned}
$$

where

$$
W(\lambda)=\sum_{p=1}^{\nu-1} w_{p} \lambda^{2 p}
$$

with

$$
w_{p}=\frac{1}{2 v_{p}(p+1)} \sum_{j=p}^{\nu-1} h_{j} v_{j} .
$$

As a result of these calculations and taking the limit as $\lambda \rightarrow \infty$, we deduce the formula

$$
-l=\beta^{2} \sum_{j=1}^{\nu-1} w_{j} v_{j-1}-\sum_{j=1}^{\nu-1} w_{j} v_{j}-1+\log \left(\frac{\beta^{2}}{4}\right),
$$

in which terms of non-constant order (which must cancel in any case) have been dropped.

Remark The relations generated by setting the non-constant terms equal to zero are equivalent to the moment conditions 1.1] (4) for the measure $\psi$ (see also [21]).

Equation (3.28) simplifies to the expression:

$$
-l=\frac{4(2 \nu-1)(2 \nu-3) ! t}{x \nu(\nu-2) !^{2}} \frac{\beta^{2 \nu}}{4^{\nu}}-1+\log \left(\frac{\beta^{2}}{4}\right) .
$$

Next, we use (3.14) to express $t$ as a function of $\beta^{2}$ and $x$ :

$$
t=-\frac{(\nu-1) !^{2} 4^{\nu-1}}{2(2 \nu-1) ! \beta^{2 \nu}}\left(\beta^{2}-4 x\right)
$$

Substituting (3.30 into 3.29) we finally have:

$$
-l=-\frac{\nu-1}{\nu}\left(\frac{\beta^{2}}{4 x}-1\right)-1+\log \left(\frac{\beta^{2}}{4}\right) .
$$

As an example, consider $\nu=2$ for which equation (3.31) becomes

$$
-l=-\frac{1}{2}\left(\frac{\beta^{2}}{4 x}-1\right)-1+\log \left(\frac{\beta^{2}}{4}\right) .
$$


3.3. Explicit calculation of $(V, \psi)$. Next we need to find an expression for $(V, \psi)$ :

$$
(V, \psi)=\frac{1}{x} \int_{-\beta}^{\beta}\left(\frac{1}{2} \lambda^{2}+t \lambda^{2 \nu}\right) h(\lambda) \sqrt{\lambda^{2}-\beta^{2}} d \lambda .
$$

Evaluating (3.32) by regarding its double as a contour integral and computing the value in terms of $v_{j}$ and $h_{j}$, we find that

$$
(V, \psi)=\frac{1}{4 x} \sum_{j=0}^{\nu-1} h_{j} v_{j+1}+\frac{v_{1}}{4 x}+\frac{t}{2 x} \sum_{j=0}^{\nu-1} h_{j} v_{j+\nu}+\frac{t v_{\nu}}{2 x} .
$$

This expression simplifies to

$$
(V, \psi)=\frac{4^{-1-\nu} t \beta^{2 \nu+2}(2 \nu) !}{x(\nu+1)(\nu-1) !^{2}}+\frac{\beta^{4}}{32 x}+\frac{24^{-2 \nu} t^{2}(2 \nu-1) !^{2} \beta^{4 \nu}}{x \nu(\nu-1) !^{4}}+\frac{4^{-1-\nu}(\nu+1) t \beta^{2 \nu+2}(2 \nu) !}{x(\nu+1) !^{2}} .
$$

As before, we simplify equation (3.34) by substituting the expression (3.30) for $t$ in terms of $\beta^{2}$ :

$$
(V, \psi)=-\frac{-8 x \beta^{2} \nu^{2}+\beta^{4} \nu^{2}-2 \beta^{4} \nu-x^{2} 16 \nu-x^{2} 16+8 \beta^{2} \nu x+\beta^{4}}{32 x(\nu+1) \nu} .
$$

When $\nu=2$, we find that

$$
(V, \psi)=\frac{1}{12} \beta^{2}-\frac{1}{192 x} \beta^{4}+\frac{x}{4}
$$

3.4. The explicit formula for $e_{0}(t)$. We can now put all of the pieces together. Evaluating the above expressions when $t=0$ (or equivalently when $\beta=2 \sqrt{x}$ ), we find $E_{0}=-3 / 4+1 / 2 \log (x)$. Collecting all the components gives

$$
e_{0}=\frac{1}{16 x^{2}} \mu\left(\beta^{2}-4 x\right)\left(\beta^{2}-4 x r\right)+\frac{1}{2} \log \left(\frac{\beta^{2}}{4 x}\right)=\eta(z-1)(z-r)+\frac{1}{2} \log (z),
$$

where

$$
\begin{aligned}
\eta & =\frac{(\nu-1)^{2}}{4 \nu(\nu+1)} \\
r & =\frac{3(\nu+1)}{\nu-1}
\end{aligned}
$$

and

$$
z=\frac{\beta^{2}}{4 x}
$$

We have proven that $e_{0}$ has an explicit representation depending only on $\nu$ (see the first part of Theorem 2.11).

When $\nu=2$ the result is

$$
e_{0}=\frac{1}{24}(z-1)(z-9)+\frac{1}{2} \log (z)
$$

Remark It is interesting to compare our expression here to a formula derived in [8]. The difference is that while our formula is more explicit, it is restricted to a 1-parameter family of times corresponding to a fixed valence of the vertices. 
3.5. Enumeration of Planar Graphs. It follows from Theorem 1.3 that the coefficients, $\kappa_{0}^{(\nu)}(j)$, of the leading order term $e_{0}(t)$ count the number of planar $2 \nu$-regular maps with $j$ vertices. We have shown that $e_{0}(t)$ can be explicitly expressed in terms of the auxiliary function $z(t)$. The latter solves the algebraic relation (3.15). So to find the Taylor Coefficients of $e_{0}(t)$, we must first find the Taylor Coefficients of $z(t)$.

We define

$$
\alpha=-c_{\nu} x^{\nu-1} t
$$

where

$$
c_{\nu}=2 \nu\left(\begin{array}{c}
2 \nu-1 \\
\nu-1
\end{array}\right)
$$

Then the polynomial relation defining $z(t)$ is

$$
1=z(t)-\alpha z(t)^{\nu}
$$

in a neighborhood of $t=0$. (We view $x$ here as a scaling parameter. When $x$ is set to 1 , we will recover the counting function.)

The $j^{t h}$ coefficient of the Taylor expansion of $z$ as a function of $\alpha$ near $0, z=\sum_{j \geq 0} \zeta_{j} \alpha^{j}$, is of course given by

$$
\zeta_{j}=\frac{1}{2 \pi i} \oint \frac{z(\alpha)}{\alpha^{j+1}} d \alpha
$$

Making the substitution $u=z(\alpha)$, and using the evaluation

$$
\frac{d z}{d \alpha}=\frac{z^{\nu}}{1-\nu \alpha z^{\nu-1}}
$$

derived by differentiating the relation (3.43), and also using (3.43) to eliminate $\alpha$, this integral becomes

$$
\frac{1}{2 \pi i} \oint_{u \sim 1} \frac{(\nu-(\nu-1) u) u^{\nu j}}{(u-1)^{j+1}} d u
$$

Applying the binomial expansion, it is then straightforward to evaluate this loop integral and find that

$$
\zeta_{j}=(1-\nu)\left(\begin{array}{c}
\nu j+1 \\
j
\end{array}\right)+\nu\left(\begin{array}{c}
\nu j \\
j
\end{array}\right)=\frac{1}{j}\left(\begin{array}{c}
\nu j \\
j-1
\end{array}\right) .
$$

We note that these coefficients are precisely the higher Catalan numbers, which play a role in a wide variety of combinatorial problems. For a discussion of these applications and their relation to the work discussed here see 22.

A similar approach will yield coefficients for the other terms in the expression (3.37) for $e_{0}$. For instance the Taylor coefficients of $\log (z(\alpha))=\sum_{j=1}^{\infty} L_{j}^{(\nu)} \alpha^{j}$ are given by

$$
L_{j}^{(\nu)}=\frac{1}{2 \pi i} \oint_{u \sim 1} \frac{\log (u)\left(1-\nu \alpha u^{\nu-1}\right) u^{j \nu}}{(u-1)^{j+1}} d u .
$$

Expanding the integrand in the vicinity of $u=1$, we can evaluate these coefficients as

$$
L_{j}^{(\nu)}=\sum_{k+\ell=j-1}\left\{(1-\nu)\left(\begin{array}{c}
\nu j \\
j
\end{array}\right)+\nu\left(\begin{array}{c}
\nu j-1 \\
j
\end{array}\right)\right\} \frac{(-1)^{k}}{k+1}=\frac{1}{j}\left(\begin{array}{c}
\nu j-1 \\
j-1
\end{array}\right)
$$

By the same method one also derives the coefficients in the expansion of the quadratic term, $(z(\alpha)-1)^{2}=$ $\sum_{j=2}^{\infty} U_{2, j}^{(\nu)} \alpha^{j}:$

$$
U_{2, j}^{(\nu)}=\frac{2}{j}\left(\begin{array}{c}
\nu j \\
j-2
\end{array}\right)
$$


Finally one may assemble all these contributions in (3.37) and substitute for $\alpha$ as in (3.42) to conclude that the Taylor coefficients of $e_{0}$ with respect to $t_{2 \nu}$ satisfy

$$
\begin{aligned}
\frac{\kappa_{0}^{(\nu)}(j)}{c_{\nu}^{j}} & =j !\left[-(r-1) \eta \zeta_{j}+\eta U_{2, j}^{(\nu)}+\frac{1}{2} L_{j}^{(\nu)}\right] \\
& =\left(-(r-1) \eta \frac{(\nu j) !}{((\nu-1) j+1) !}+\eta \frac{2(j-1)(\nu j) !}{((\nu-1) j+2) !}+\frac{1}{2} \frac{(\nu j-1) !}{((\nu-1) j+2) !}\right) .
\end{aligned}
$$

After simplifying this equation we get

$$
\kappa_{0}^{(\nu)}(j)=c_{\nu}^{j} \frac{(\nu j-1) !}{((\nu-1) j+2) !} .
$$

This establishes the second half of Theorem 2.1

\section{Continuum Toda Equations}

We will show that the Toda Lattice equations at level $2 \nu$, with the initial conditions $b_{k}=\sqrt{k}$, possess a continuum limit under an appropriate scaling. These equations will lead to a description of the evolution of the asymptotic partition function coefficients, $e_{g}(t)$. More precisely, in Section 4.1 we establish Theorem 2.2 and in subsection 4.2 we establish Theorem 2.7. To this end, we first review some basic facts about the Toda lattice equations.

The Toda Lattice Hierarchy is given by the differential equations

$$
\frac{d L}{d \xi_{i}}=\left[B_{i}(L), L\right],
$$

where $L$ is a symmetric tridiagonal matrix of the form

$$
L=\left(\begin{array}{cccccc}
a_{1} & b_{1} & 0 & 0 & 0 & \ldots \\
b_{1} & a_{2} & b_{2} & 0 & 0 & \ldots \\
0 & b_{2} & a_{3} & b_{3} & 0 & \ldots \\
0 & 0 & b_{3} & a_{4} & b_{4} & \ldots \\
\vdots & \vdots & \vdots & \vdots & \vdots &
\end{array}\right),
$$

and $B_{i}(L)=\left(L^{i}\right)_{+}-\left(L^{i}\right)_{-}$where the plus subscript denotes upper triangular projection and the minus subscript denotes lower triangular projection.

We briefly recall the notion of tau functions in terms of which the solution of the Toda Lattice equations at different time parameters can be expressed. Let $L_{0}$ be a semi-infinite tri-diagonal matrix of the type that arises as a 3-term recurrence relation for orthogonal polynomials with exponential weight. The dependence of the $j^{\text {th }}$ tau function on the $\xi_{i}$ is given by

$$
\tau_{j}\left(\xi_{1}, \ldots, \xi_{v} ; L_{0}\right)=\left(\operatorname{det}\left[M_{j}^{2}\right]\right)^{1 / 2},
$$

where $M=\exp \left(\sum_{i=1}^{v} \xi_{i} L_{0}^{i}\right)$ and $M_{j}$ is the $j \times j$ upper left block of $M$ in the basis of orthogonal polynomials with respect to $d \mu$. From the matrix factorization method of solving the Toda equations 18 one finds that

$$
b_{j}(\vec{\xi})=\frac{\tau_{j+1}(\vec{\xi}) \tau_{j-1}(\vec{\xi})}{\tau_{j}(\vec{\xi})^{2}} b_{j}(0) .
$$

Furthermore, from (4.4) and the differential equations for the $\xi_{1}$-Toda flow, one can deduce that

$$
b_{j}(\vec{\xi})=\sqrt{\frac{1}{2} \frac{d^{2}}{d \xi_{1}^{2}} \log \left(\tau_{j}(\vec{\xi})\right)} .
$$

The representations (4.4) and (4.5) of $b_{j}$ in terms of tau functions are referred to as Hirota relations, familiar from the theory of soliton equations. 
Finally, through the use of Hankel determinants, the tau function may be related to the partition function, $Z_{k}=Z_{k}^{(k)}$. Recall that [25] the Hankel determinant associated to the weight $d \omega(\lambda)=\exp \left(\sum_{i=1}^{v} \xi_{i} \lambda^{i}\right) d \lambda$ is defined as follows

$$
H_{k}(\vec{\xi})=\left|\begin{array}{cccc}
c_{0} & c_{1} & \cdots & c_{k-1} \\
c_{1} & c_{2} & \cdots & c_{k} \\
\vdots & \vdots & \ddots & \vdots \\
\vdots & \vdots & \ddots & \vdots \\
c_{k-1} & c_{k} & \cdots & c_{2 k-2}
\end{array}\right|
$$

where $c_{j}=\int_{\mathbb{R}} \lambda^{j} d \omega(\lambda)$ are the moments of $d \omega$. There follows the relation

$$
b_{j}^{2}(\vec{\xi})=\frac{H_{j+1}(\vec{\xi}) H_{j-1}(\vec{\xi})}{H_{j}(\vec{\xi})^{2}} .
$$

The partition function may be obtained from the Hankel determinant by a rescaling of the time parameters (see, for example, [15. Equation (1.29)]). Consequently one can relate the partition function to the tau function, in the case of interest to us, as

$$
\tau_{k}\left(\xi_{1}, \xi_{2 \nu} ; L_{0}\right)^{2}=\frac{Z_{k}\left(-\xi_{1},-2 k^{\nu-1} \xi_{2 \nu}\right)}{Z_{k}(0)},
$$

where $L_{0}$ is the semi-infinite tridiagonal matrix with $b_{k}=\sqrt{k}, a_{k} \equiv 0$, and where $\xi=\xi_{2 \nu}$. For this initial condition, the evolution of $L$ under the even $2 \nu$-flow preserves the vanishing of $a_{k}$. It will be natural to study this Toda evolution in terms of the self-similar variable

$$
s=2 k^{\nu-1} \xi .
$$

We will start from the unscaled partition function (1.1):

$$
\begin{aligned}
& Z_{1}^{(k)}\left(-\xi_{1},-\xi\right)= \\
& \int \cdots \int \exp \left\{-\sum_{j=1}^{k}\left(\frac{1}{2} \lambda_{j}^{2}-\xi \lambda_{j}^{2 \nu}-\xi_{1} \lambda_{j}\right)\right\} \mathcal{V}(\lambda) d^{k} \lambda, \\
& \text { where } \mathcal{V}(\lambda)=\prod_{j \neq \ell}\left|\lambda_{j}-\lambda_{\ell}\right| .
\end{aligned}
$$

Using the Hirota relations, (4.4) and (4.5), we have the following Proposition.

Proposition 4.1. The Toda recurrence coefficients may be represented as follows.

$$
b_{k}^{2}=\left.\frac{1}{2 k} \frac{d^{2}}{d t_{1}^{2}} \log \left[Z_{k}\left(t_{1},-s\right)\right]\right|_{t_{1}=0}, \quad \text { where } s \leq 0 .
$$

In addition, the following asymptotic expansion holds true.

$$
b_{k}^{2}=k \sum_{g \geq 0} z_{g}(s) \frac{1}{k^{2 g}},
$$

where $z_{g}(s)$ is an analytic function of $s$ in a neighborhood of $s=0$, and

$$
z_{0}(0)=1, z_{g}(0)=0, g>0 \text {. }
$$

To prove this proposition we scale the eigenvalues in the unscaled partition function as $\lambda_{j}=N^{1 / 2} \tilde{\lambda}_{j}$ under which

$$
\begin{aligned}
Z_{1}^{(k)} & =N^{k^{2} / 2} \int \cdots \int \exp \left(-N\left\{\sum_{j=1}^{k}\left(\frac{1}{2} \tilde{\lambda}_{j}^{2}-\xi N^{\nu-1} \tilde{\lambda}_{j}^{2 \nu}-\frac{\xi_{1}}{\sqrt{N}} \tilde{\lambda}_{j}\right)\right\}\right) \mathcal{V}(\tilde{\lambda}) d^{k} \tilde{\lambda} \\
& =N^{k^{2} / 2} Z_{N}^{(k)}\left(-\frac{\xi_{1}}{\sqrt{N}},-\xi N^{\nu-1}\right) .
\end{aligned}
$$


We observe that the Toda variables associated to our random matrix ensemble with the corresponding $\mathrm{N}$ scaling have the following representation:

$$
\begin{aligned}
b_{k}^{2} & =\frac{1}{2} \frac{d^{2}}{d \xi_{1}^{2}} \log Z_{1}^{(k)} \\
& =\frac{1}{2} \frac{d^{2}}{d \xi_{1}^{2}} \log \left[Z_{N}^{(k)}\left(-\frac{\xi_{1}}{\sqrt{N}},-\xi N^{\nu-1}\right)\right] \\
& =\left.\frac{1}{2 N} \frac{d^{2}}{d t_{1}^{2}} \log \left[Z_{N}^{(k)}\left(t_{1}, t\right)\right]\right|_{t=-2 \xi N^{\nu-1}, t_{1}=-\frac{\xi_{1}}{\sqrt{N}}}
\end{aligned}
$$

We next define a spatial scale through the ratio between $k$ and $N$ : set $k=x N$. If we furthermore introduce the scaling $\tilde{\lambda}_{j}=\sqrt{x} \hat{\lambda}_{j}$, then we can rewrite

$$
\begin{aligned}
Z_{N}^{(k)}\left(t_{1}, t\right) & =x^{k^{2} / 2} \int \cdots \int \exp \left(-k\left\{\sum_{j=1}^{k}\left(\frac{1}{2} \hat{\lambda}_{j}^{2}+t x^{\nu-1} \hat{\lambda}_{j}^{2 \nu}+\frac{t_{1}}{\sqrt{x}} \hat{\lambda}_{j}\right)\right\}\right) \mathcal{V}(\hat{\lambda}) d^{k} \hat{\lambda} \\
& =x^{k^{2} / 2} Z_{k}^{(k)}\left(\frac{t_{1}}{\sqrt{x}}, t x^{\nu-1}\right)
\end{aligned}
$$

Putting all the above together, the natural variable to consider for this continuum limit of the Toda Lattice Equations is

$$
\frac{1}{k} b_{k}^{2}=\left.\frac{1}{2 k^{2}} \frac{d^{2}}{d t_{1}^{2}} \log \left[Z_{k}^{(k)}\left(t_{1},-s\right)\right]\right|_{t_{1}=-\frac{1}{\sqrt{k}} \xi_{1}, s=2 k^{\nu-1} \xi}
$$

One may conclude directly from the main theorem of [15] that $\frac{1}{k^{2}} \log Z_{k}^{(k)}$ has a full asymptotic expansion in powers of $k^{-2}$, uniformly valid in an appropriate $\left(t_{1},-s\right)$ domain which includes arbitrary negative $s$ and simultaneously an open neighborhood of $t_{1}=0$. On this domain the asymptotic expansion of derivatives may be calculated via term by term differentiation of the original asymptotic expansion. Moreover, the coefficients in this expansion are complex analytic in a neighborhood of $(0,0)$. It follows that

$$
\left.\frac{1}{2 k^{2}} \frac{d^{2}}{d t_{1}^{2}} \log \left[Z_{k}^{(k)}\left(t_{1},-s\right)\right]\right|_{t_{1}=0}
$$

has a full asymptotic expansion in powers of $k^{-2}$ uniformly valid for negative $s$ and whose coefficients are analytic in a complex neighborhood of $s=0$.

4.1. The Continuum Toda Hierarchy. In this subsection we prove Theorem 2.2.

We begin with the Toda lattice systems (4.1) introduced earlier. It will prove convenient to transform this to the so-called Kostant-Toda lattice form [14] where L is asymmetric tri-diagonal but in which the $b_{k}^{2}$ are the fundamental variables. This is achieved by conjugating $L$ in (4.1) by the diagonal matrix

$$
D=\left(\begin{array}{cccccc}
1 & 0 & 0 & 0 & 0 & \ldots \\
0 & b_{1}^{-1} & 0 & 0 & 0 & \ldots \\
0 & 0 & \left(b_{1} b_{2}\right)^{-1} & 0 & 0 & \ldots \\
0 & 0 & 0 & \left(b_{1} b_{2} b_{3}\right)^{-1} & & \ldots \\
\vdots & \vdots & \vdots & \vdots & \ddots &
\end{array}\right)
$$


The fundamental matrix variable for the Kostant-Toda system is

$$
\mathcal{L}=D^{-1} L D=\left(\begin{array}{cccccc}
0 & 1 & 0 & 0 & 0 & \ldots \\
b_{1}^{2} & 0 & 1 & 0 & 0 & \ldots \\
0 & b_{2}^{2} & 0 & 1 & 0 & \ldots \\
0 & 0 & b_{3}^{2} & 0 & 1 & \ddots \\
\vdots & \vdots & \ddots & \ddots & \ddots &
\end{array}\right)
$$

Because $D$ is diagonal it is straightforward to see that the RHS of (4.1) transforms as

$$
D^{-1}\left[B_{2 \nu}(L), L\right] D=\left[D^{-1} B_{2 \nu}(L) D, D^{-1} L D\right]=\left[B_{2 \nu}(\mathcal{L}), \mathcal{L}\right] .
$$

One also has

$$
\mathcal{L}_{\xi}=\left(D^{-1} L D\right)_{\xi}=D^{-1} L_{\xi} D+\left[\mathcal{L}, D^{-1} D_{\xi}\right] .
$$

Combining this observation with (4.20) in (4.1) we see that the Lax equation transforms to

$$
\mathcal{L}_{\xi}+\left[D^{-1} D_{\xi}, \mathcal{L}\right]=\left[B_{2 \nu}(\mathcal{L}), \mathcal{L}\right] .
$$

By direct calculation one can check that

$$
\left[D^{-1} D_{\xi}, \mathcal{L}\right]=-\frac{1}{2} \mathcal{L}_{\xi}+\left[D^{-1} D_{\xi}, \epsilon\right]
$$

where

$$
\epsilon=\left(\begin{array}{cccccc}
0 & 1 & 0 & 0 & 0 & \cdots \\
0 & 0 & 1 & 0 & 0 & \cdots \\
0 & 0 & 0 & 1 & 0 & \cdots \\
0 & 0 & 0 & 0 & 1 & \ddots \\
\vdots & \vdots & \ddots & \ddots & \ddots &
\end{array}\right) .
$$

Using this observation the Lax equation (4.21) becomes

$$
\frac{1}{2} \mathcal{L}_{\xi}=\left[B_{2 \nu}(\mathcal{L}), \mathcal{L}\right]-\left[D^{-1} D_{\xi}, \epsilon\right]
$$

Since the LHS is lower triangular, we may reduce this equation to

$$
\frac{1}{2} \mathcal{L}_{\xi}=\left[B_{2 \nu}(\mathcal{L}), \mathcal{L}\right]_{-} .
$$

Since $\mathcal{L}$ is tri-diagonal, the RHS may be further simplified as

$$
\left[B_{2 \nu}(\mathcal{L}), \mathcal{L}\right]_{-}=\left[\mathcal{L}_{+}^{2 \nu}-\mathcal{L}_{-}^{2 \nu}, \mathcal{L}\right]_{-}=-\left[\mathcal{L}_{-}^{2 \nu}, \mathcal{L}\right]_{-} .
$$

Finally, since $\mathcal{L}=\mathcal{L}_{-}+\epsilon$ with $\mathcal{L}_{-}$supported only on the first lower diagonal, it follows that $\mathcal{L}_{\xi}$ is only supported on the first lower diagonal and therefore we may use the following final form of our Lax equation:

$$
\frac{1}{2} \mathcal{L}_{\xi}=\left[\epsilon, \mathcal{L}_{-}^{2 \nu}\right]_{(-1)}
$$

where the subscript $(-1)$ denotes projection onto the first lower subdiagonal. At the level of the matrix entries $b_{k}^{2}$ this system of equations becomes

$$
\frac{1}{2} \frac{d b_{k}^{2}}{d \xi}=\left(\mathcal{L}^{2 \nu}\right)_{k+2, k}-\left(\mathcal{L}^{2 \nu}\right)_{k+1, k-1}
$$

where,

$$
\left(\mathcal{L}^{2 \nu}\right)_{k+1, k-1} \sum_{i_{1}, i_{2}, \ldots, i_{2 \nu+1} ;\left|i_{j+1}-i_{j}\right|=1 ; i_{1}=k+1, i_{2 \nu+1}=k-1} \mathcal{L}_{k+1, i_{2}} \mathcal{L}_{i_{2}, i_{3}} \ldots \mathcal{L}_{i_{2 \nu}, k-1} .
$$

as stated in (2.2) and (2.3) .

Note that in (4.27) the sum may be viewed as being taken over walks, $w$, from $\mathrm{k}+1$ to $\mathrm{k}-1$ of length $2 \nu$. We will sometimes represent a walk $w$ as a $2 \nu$-vector of $\pm 1^{\prime} s$. It is clear that such a walk has $\nu+1$ "downturns" (vector entries of -1) and $\nu-1$ "upturns" (vector entries of +1 ). Therefore, specifying the locations of the 
downturns determines $w$. From this we see that the set of all such walks, $\{w\}$ is in one-to-one correspondence with all choices of $\nu+1$ numbers (the downturn locations), $\left\{j_{1}<j_{2}<\cdots<j_{\nu+1}\right\}$ from $\{1, \ldots, 2 \nu\}$ :

$$
\sharp\{w\}=\left(\begin{array}{c}
2 \nu \\
\nu+1
\end{array}\right) \text {. }
$$

Thus (4.27) becomes

$$
\sum_{\{w\}} b_{k+\ell_{1}(w)}^{2} b_{k+\ell_{2}(w)}^{2} \cdots b_{k+\ell_{\nu+1}(w)}^{2},
$$

where

$$
\ell_{m}(w)=j_{m}-2 m+1 .
$$

Some explanation is perhaps necessary here. The sum appearing in (4.28) is taken over all choices of $\nu+1$ numbers, and each such choice is denoted by a member $w$ of the set $\{w\}$. As described above, each $w$ may be interpreted as a walk on the integer lattice, from $k+1$ to $k-1$ of length $2 \nu$. Since the structure of the walks and all associated counting is independent of the value of $\mathrm{k}$, we will from now on, for simplicity, take $\mathrm{k}=0$. The quantity $\ell_{m}(w)$ is defined to be the location on the integer lattice after the $m$ th downturn. It is now straightforward to compute that, given a walk with downturns at $j_{1}, \ldots, j_{\nu+1}$, after $m$ downturns, there have been $j_{m}-m$ upward steps, and $m$ downward steps. Since the starting position is +1 , (4.29) follows.

Inserting these reductions into the Toda equations (4.26) we have

$$
\frac{1}{2}\left(b_{k}^{2}\right)_{\xi}=\sum_{\{w\}}\left[\prod_{m=1}^{\nu+1} b_{k+\ell_{m}(w)+1}^{2}-\prod_{m=1}^{\nu+1} b_{k+\ell_{m}(w)}^{2}\right] .
$$

By Proposition 4.1]

$$
b_{k}^{2}(\xi)=b_{k}^{2}\left(\frac{s_{k}}{2 k^{\nu-1}}\right)=\widehat{b}_{k}^{2}\left(s_{k}\right)=k\left(\left.\frac{1}{2 k^{2}} \frac{d^{2}}{d t_{1}^{2}} \log \left(Z_{k}\left(t_{1},-s_{k}\right)\right)\right|_{t_{1}=0}\right),
$$

and the differential equation (4.30) becomes

$$
k^{\nu-1} \frac{d}{d s_{k}} \widehat{b}_{k}^{2}\left(s_{k}\right)=\sum_{\{w\}}\left[\prod_{m=1}^{\nu+1} \widehat{b}_{k+\ell_{m}(w)+1}^{2}\left(s_{k+\ell_{m}+1}\right)-\prod_{m=1}^{\nu+1} \widehat{b}_{k+\ell_{m}(w)}^{2}\left(s_{k+\ell_{m}}\right)\right] .
$$

¿From 4.31), and the asymptotic expansion satisfied by the partition function appearing therein, one sees that for any fixed integer $\ell$, the quantity $(k+\ell)^{-1} \widehat{b}_{k+\ell}^{2}\left(s_{k+\ell}\right)$ possesses an asymptotic expansion in even inverse powers of $(k+\ell)$, which we express in the following form:

$$
\widehat{b}_{k+\ell}^{2}\left(s_{k+\ell}\right)=k \sum_{g \geq 0} z_{g}\left(s_{k}\left(1+\frac{\ell}{k}\right)^{\nu-1}\right)\left(1+\frac{\ell}{k}\right)^{1-2 g} k^{-2 g} .
$$

\section{Fundamental Asymptotic Principle: Recall that}

i: the asymptotic expansion satisfied by the partition function is differentiable term by term

ii: the coefficients $z_{g}(\bullet)$ are analytic functions of $\bullet$.

Therefore, the terms appearing in the summation in (4.33) may be Taylor expanded (note that $\ell / k$ is asymptotically small) and the resulting multiple series may be re-summed. One may convince oneself of this as follows. One truncates the expansion (4.33) at order $k^{1-2 h}$, and observes that the error is $O\left(k^{-1-2 h}\right)$. Then one computes Taylor expansions to order $k^{-2 h}$ of all quantities appearing in the (now finite) sum, and observes that the error is $O\left(k^{-1-2 h}\right)$.

Following this principle, we now introduce the scheme $f(s, w)$ :

$$
f(s, w)=\sum_{g \geq 0} z_{g}\left(s_{k} w^{\nu-1}\right) w^{1-2 g} k^{-2 g} .
$$


Using this scheme, the differential equation (4.32) becomes:

$$
\begin{aligned}
\left.\frac{d}{d s} f(s, w)\right|_{w=1} & =\sum_{\{w\}} k f^{\nu+1}\left\{\prod_{m=1}^{\nu+1}\left[1+\frac{f_{w}}{f}\left(\frac{\ell_{m}+1}{k}\right)+\frac{f_{w^{(2)}}}{2 f}\left(\frac{\ell_{m}+1}{k}\right)^{2}+\cdots+\frac{f_{w^{(h)}}}{h ! f}\left(\frac{\ell_{m}+1}{k}\right)^{h}+\cdots\right]\right. \\
& =\sum_{\{w\}}\left\{(\nu+1) f^{\nu} f_{w}+\mathcal{O}\left(\frac{1}{k^{2}}\right)\right\} \\
& =(\nu+1)\left(\begin{array}{c}
2 \nu \\
\nu+1
\end{array}\right) f^{\nu} f_{w}+\mathcal{O}\left(\frac{1}{k^{2}}\right),
\end{aligned}
$$

where we note that $(\nu+1)\left(\begin{array}{c}2 \nu \\ \nu+1\end{array}\right)=2 \nu\left(\begin{array}{c}2 \nu-1 \\ \nu-1\end{array}\right)=c_{\nu}$ as claimed in the statement of Theorem 2.2

From the expansion (4.34) one may now confirm that the higher order terms in the expansion of the RHS have the form described in Theorem 2.2 The justification for the form of the coefficient $d_{V}^{(\nu, g)}$ stated there requires some further explanation which we now provide.

Given the explicit determination (4.29) of the $\ell_{m}$ in terms of the walks, it is natural to try to calculate $d_{V}^{(\nu, g)}$ by expressing this combinatorial quantity in terms of expectations with respect to the induced probability distribution on the family of random walks $\{w\}$ conditioned to begin at +1 and end at -1 . To this end we observe that given an ordered sequence of numbers $m_{1}<m_{2}<\cdots<m_{r}$ selected from the set $\{1, \ldots, \nu+1\}$, and another ordered sequence $i_{1}<\cdots<i_{r}$ selected from $\{1, \ldots, 2 \nu\}$,

$$
\begin{aligned}
& \sharp\left\{w \mid m_{j}^{\text {th }} \text { downturn of } w \text { occurs at position } i_{j}, j=1, \ldots, r\right\} \\
= & \left(\begin{array}{c}
i_{1}-1 \\
m_{1}-1
\end{array}\right)\left(\begin{array}{c}
i_{2}-i_{1}-1 \\
m_{2}-m_{1}-1
\end{array}\right) \cdots\left(\begin{array}{c}
i_{r}-i_{r-1}-1 \\
m_{r}-m_{r-1}-1
\end{array}\right)\left(\begin{array}{c}
2 \nu-i_{r} \\
\nu+1-m_{r}
\end{array}\right) .
\end{aligned}
$$

These joint probability distributions are fundamental for the count we will now describe. 
One sees directly from expanding the products in (4.35), that

$$
\begin{aligned}
& d_{V}^{(\nu, g)}=\sum_{\{w\}} \frac{1}{\prod_{j=1}^{2 g+1} r_{j}(V) !} \sum_{\substack{m_{1}, \ldots, m_{\rho(V)} \\
\text { di=1 }}}\left(\prod_{i=1}^{\rho(V)}\left(\ell_{m_{i}}+1\right)^{\left|V_{i}\right|}-\prod_{i=1}^{\rho(V)}\left(\ell_{m_{i}}\right)^{\left|V_{i}\right|}\right) \\
& \in\{1, \ldots, \nu+1\} \\
& =\frac{1}{\prod_{j=1}^{2 g+1} r_{j}(V) !} \sum_{\substack{m_{1}, \ldots, m_{\rho(V)} \\
\sum_{w w\}}}}\left(\prod_{i=1}^{\rho(V)}\left(\ell_{m_{i}}+1\right)^{\left|V_{i}\right|}-\prod_{i=1}^{\rho(V)}\left(\ell_{m_{i}}\right)^{\left|V_{i}\right|}\right) \\
& \text { distinct } \\
& \in\{1, \ldots, \nu+1\} \\
& =\frac{1}{\prod_{j=1}^{2 g+1} r_{j}(V) !} \sum_{\sigma \in \mathcal{S}_{\rho(V)}} \sum_{\substack{m_{1}<\cdots<m_{\rho(V)} \\
\in\{1, \ldots, \nu+1\}}} \sum_{\{w\}}\left(\prod_{i=1}^{\rho(V)}\left(\ell_{m_{\sigma(i)}}+1\right)^{\left|V_{i}\right|}-\prod_{i=1}^{\rho(V)}\left(\ell_{m_{\sigma(i)}}\right)^{\left|V_{i}\right|}\right) \\
& =\frac{1}{\prod_{j=1}^{2 g+1} r_{j}(V) !} \sum_{\sigma \in \mathcal{S}_{\rho(V)}} \sum_{1 \leq i_{1} \cdots<i_{\rho(V)} \leq 2 \nu} \sum_{m_{1}<\cdots<m_{\rho(V)}} \\
& \in\{1, \ldots, \nu+1\} \\
& \left(\prod_{j=1}^{\rho(V)}\left(i_{j}-2 m_{j}+2\right)^{\left|V_{\sigma(j)}\right|}\left(\begin{array}{c}
i_{1}-1 \\
m_{1}-1
\end{array}\right)\left(\begin{array}{c}
i_{2}-i_{1}-1 \\
m_{2}-m_{1}-1
\end{array}\right) \cdots\left(\begin{array}{c}
i_{\rho(V)}-i_{\rho(V)-1}-1 \\
m_{\rho(V)}-m_{\rho(V)-1}-1
\end{array}\right)\left(\begin{array}{c}
2 \nu-i_{\rho(V)} \\
\nu+1-m_{\rho(V)}
\end{array}\right)-\right. \\
& \left.\prod_{j=1}^{\rho(V)}\left(i_{j}-2 m_{j}+1\right)^{\left|V_{\sigma(j)}\right|}\left(\begin{array}{c}
i_{1}-1 \\
m_{1}-1
\end{array}\right)\left(\begin{array}{c}
i_{2}-i_{1}-1 \\
m_{2}-m_{1}-1
\end{array}\right) \cdots\left(\begin{array}{c}
i_{\rho(V)}-i_{\rho(V)-1}-1 \\
m_{\rho(V)}-m_{\rho(V)-1}-1
\end{array}\right)\left(\begin{array}{c}
2 \nu-i_{\rho(V)} \\
\nu+1-m_{\rho(V)}
\end{array}\right)\right)
\end{aligned}
$$

where in the last equality we have applied (4.29) and (4.36).

The formula for $d_{V}^{(\nu, g)}$ given in Theorem 2.2 now follows from the next proposition.

Proposition 4.2. The inner-most summation may be re-expressed in terms of the following combinatorial coefficients:

$$
\begin{aligned}
& \text { coeff of } x^{\nu-r+1} \text { in } \\
& \left.\left(\prod_{n=1}^{r}\left(i_{n}-2 \sum_{s=1}^{n}\left(1+x_{s} \frac{\partial}{\partial x_{s}}\right)+c\right)^{\alpha_{n}}\right) \cdot\left(1+x_{1}\right)^{i_{1}-1} \cdots\left(1+x_{r}\right)^{i_{r}-i_{r-1}-1}\left(1+x_{r+1}\right)^{2 \nu-i_{r}}\right|_{x_{\mu}=x} \\
& =\sum_{m_{1}<\cdots<m_{\rho(V)}} \\
& \in\{1, \ldots, \nu+1\} \\
& \prod_{n=1}^{r}\left(i_{n}-2 m_{n}+c\right)^{\alpha_{n}}\left(\begin{array}{c}
i_{1}-1 \\
m_{1}-1
\end{array}\right)\left(\begin{array}{c}
i_{2}-i_{1}-1 \\
m_{2}-m_{1}-1
\end{array}\right) \cdots\left(\begin{array}{c}
i_{r}-i_{r-1}-1 \\
m_{r}-m_{r-1}-1
\end{array}\right)\left(\begin{array}{c}
2 \nu-i_{r} \\
\nu+1-m_{r}
\end{array}\right) .
\end{aligned}
$$


To prove this we start with the LHS which, after expanding binomially, becomes

$$
\begin{aligned}
& \text { coeff of } x^{\nu-r+1} \text { in }\left(\prod_{n=1}^{r}\left(i_{n}-2 \sum_{s=1}^{n}\left(1+x_{s} \frac{\partial}{\partial x_{s}}\right)+c\right)^{\alpha_{n}}\right) \cdot \sum_{s_{1}=0}^{i_{1}-1} \sum_{s_{2}=0}^{i_{2}-i_{1}-1} \cdots \sum_{s_{r}=0}^{i_{r}-i_{r-1}-1} \sum_{s_{r+1}=0}^{2 \nu-i_{r}} \\
& \left.\left(\begin{array}{c}
i_{1}-1 \\
s_{1}
\end{array}\right)\left(\begin{array}{c}
i_{2}-i_{1}-1 \\
s_{2}
\end{array}\right) \cdots\left(\begin{array}{c}
i_{r}-i_{r-1}-1 \\
s_{r}
\end{array}\right)\left(\begin{array}{c}
2 \nu-i_{r} \\
s_{r+1}
\end{array}\right) x_{1}^{s_{1}} x_{2}^{s_{2}} \cdots x_{r}^{s_{r}} x_{r+1}^{s_{r+1}}\right|_{x_{\mu}=x} \\
& =\text { coeff of } x^{\nu-r+1} \text { in } \sum_{s_{1}=0}^{i_{1}-1} \sum_{s_{2}=0}^{i_{2}-i_{1}-1} \cdots \sum_{s_{r}=0}^{i_{r}-i_{r-1}-1} \sum_{s_{r+1}=0}^{2 \nu-i_{r}} \prod_{n=1}^{r}\left(i_{n}-2 n+c-2\left(s_{1}+\cdots+s_{n}\right)\right)^{\alpha_{n}} \text {. } \\
& \left.\left(\begin{array}{c}
i_{1}-1 \\
s_{1}
\end{array}\right)\left(\begin{array}{c}
i_{2}-i_{1}-1 \\
s_{2}
\end{array}\right) \cdots\left(\begin{array}{c}
i_{r}-i_{r-1}-1 \\
s_{r}
\end{array}\right)\left(\begin{array}{c}
2 \nu-i_{r} \\
s_{r+1}
\end{array}\right) x_{1}^{s_{1}} x_{2}^{s_{2}} \cdots x_{r}^{s_{r}} x_{r+1}^{s_{r+1}}\right|_{x_{\mu}=x} \\
& =\operatorname{coeff} \text { of } x^{\nu-r+1} \text { in } \sum_{s_{1}=0}^{i_{1}-1} \sum_{s_{2}=0}^{i_{2}-i_{1}-1} \cdots \sum_{s_{r}=0}^{i_{r}-i_{r-1}-1} \sum_{s_{r+1}=0}^{2 \nu-i_{r}} \prod_{n=1}^{r}\left(i_{n}-2 n+c-2\left(s_{1}+\cdots+s_{n}\right)\right)^{\alpha_{n}} \text {. } \\
& \left(\begin{array}{c}
i_{1}-1 \\
s_{1}
\end{array}\right)\left(\begin{array}{c}
i_{2}-i_{1}-1 \\
s_{2}
\end{array}\right) \cdots\left(\begin{array}{c}
i_{r}-i_{r-1}-1 \\
s_{r}
\end{array}\right)\left(\begin{array}{c}
2 \nu-i_{r} \\
s_{r+1}
\end{array}\right) x^{s_{1}+\cdots+s_{r+1}} \\
& =\sum_{s_{1}+\cdots+s_{r+1=\nu-r+1}} \prod_{n=1}^{r}\left(i_{n}-2 n+c-2\left(s_{1}+\cdots+s_{n}\right)\right)^{\alpha_{n}} . \\
& \left(\begin{array}{c}
i_{1}-1 \\
s_{1}
\end{array}\right)\left(\begin{array}{c}
i_{2}-i_{1}-1 \\
s_{2}
\end{array}\right) \cdots\left(\begin{array}{c}
i_{r}-i_{r-1}-1 \\
s_{r}
\end{array}\right)\left(\begin{array}{c}
2 \nu-i_{r} \\
s_{r+1}
\end{array}\right)
\end{aligned}
$$

where in the first equality we use the fact that the monomials in $x_{1}, \ldots, x_{r+1}$ are each simultaneous eigenfunctions for all the differential operators $i_{n}-2 \sum_{s=1}^{n}\left(1+x_{s} \frac{\partial}{\partial x_{s}}\right)+c$ and therefore the action of the product of these operators on each eigenfunction may be replaced by the product of the corresponding eigenvalues times this function.

We next make the following change of variables in the summations

$$
\begin{aligned}
s_{1} & =m_{1}-1 \\
s_{2} & =m_{2}-m_{1}-1 \\
& \vdots \\
s_{k} & =m_{k}-m_{k-1}-1 \\
& \vdots \\
s_{r} & =m_{r}-m_{r-1}-1
\end{aligned}
$$

which then requires that

$$
s_{r+1}=\nu-r+1-\left(s_{1}+\cdots+s_{r}\right)=\nu-r+1-\left(m_{r}-r\right)=\nu+1-m_{r}
$$

and this completes the proof of the proposition.

4.2. Differential Equations for $e_{g}(t)$. We will now show that there are differential equations whose solutions determine $e_{g}(-s)$ written in terms of the functions $z_{g}(s)$. We will show that these differential equations can be used to derive expressions for the Taylor Coefficients of $e_{g}(-s), \kappa_{g}^{(\nu)}(j)$, and verify Theorem 2.5

Recall the Hirota relation (4.4). Restricted to the level $2 \nu$-time flow this becomes

$$
b_{k}(\xi)^{2}=\frac{\tau_{k+1}(\xi)^{2} \tau_{k-1}(\xi)^{2}}{\tau_{k}(\xi)^{4}} b_{k}(0)^{2}
$$

and is related to the partition function (1.1) through

$$
\tau_{k}(\xi)^{2}=\frac{Z_{k}\left(-2 \xi k^{\nu-1}\right)}{Z_{k}(0)}
$$


The logarithm of (4.37) produces a hierarchy of difference equations (discrete Hirota relations): Setting $s_{k}=2 k^{\nu-1} \xi$ in order to write

$$
\tau_{k}(\xi)^{2}=\frac{Z_{k}\left(-s_{k}\right)}{Z_{k}(0)}
$$

and applying a logarithm to both sides of equation (4.37) one obtains

$$
\log \left(\frac{Z_{k+1}\left(-s_{k+1}\right)}{Z_{k+1}(0)}\right)-2 \log \left(\frac{Z_{k}\left(-s_{k}\right)}{Z_{k}(0)}\right)+\log \left(\frac{Z_{k-1}\left(-s_{k-1}\right)}{Z_{k-1}(0)}\right)=\log \left(b_{k}(\xi)^{2}\right)-\log \left(b_{k}(0)^{2}\right) .
$$

We will next describe a continuum limit of these discrete equations which will generate a hierarchy of differential equations for the functions $e_{g}\left(t_{2 \nu}\right)$ which appear in the asymptotic expansion (1.7).

If we shift $k \mapsto k \pm 1$, then $s_{k}$ shifts: $s_{k} \mapsto s_{k \pm 1}=s_{k}(1 \pm 1 / k)^{\nu-1}$. With this substitution the left hand side of (4.39) becomes

$$
\log \left(\frac{Z_{k+1}\left(-s(1+1 / k)^{\nu-1}\right)}{Z_{k+1}(0)}\right)-2 \log \left(\frac{Z_{k}(-s)}{Z_{k}(0)}\right)+\log \left(\frac{Z_{k-1}\left(-s(1-1 / k)^{\nu-1}\right)}{Z_{k-1}(0)}\right) .
$$

Note that from Theorem 1.7 each term in 4.40) possesses an asymptotic expansion of the form

$$
\log \left(\frac{Z_{k+r}\left(-s(1+r / k)^{\nu-1}\right)}{Z_{k+r}(0)}\right)=\sum_{g \geq 0} k^{2-2 g}\left(1+\frac{r}{k}\right)^{2-2 g} e_{g}\left(-s\left(1+\frac{r}{k}\right)^{\nu-1}\right),
$$

with $r=-1,0,1$.

The expression (4.40) is a centered difference. We utilize a lemma describing centered difference expansions:

Lemma 4.3. If $\mathcal{G}$ is an analytic function, then

$$
\mathcal{G}(1+z)-2 \mathcal{G}(1)+\mathcal{G}(1-z)=\sum_{n \geq 1} \frac{2 z^{2 n}}{(2 n) !} \mathcal{G}^{(2 n)}(1)
$$

as $z \rightarrow 0$.

To apply this lemma we note that (4.40) has an asymptotic expansion of the form

$$
\begin{gathered}
\log \left(\frac{Z_{k+1}\left(-s(1+1 / k)^{\nu-1}\right)}{Z_{k+1}(0)}\right)-2 \log \left(\frac{Z_{k}(-s)}{Z_{k}(0)}\right)+\log \left(\frac{Z_{k-1}\left(-s(1-1 / k)^{\nu-1}\right)}{Z_{k-1}(0)}\right) \\
=\sum_{g \geq 0} k^{2-2 g}\left[\mathcal{G}_{g}\left(1-\frac{1}{k}\right)-2 \mathcal{G}_{g}(1)+\mathcal{G}_{g}\left(1+\frac{1}{k}\right)\right]
\end{gathered}
$$

where

$$
\mathcal{G}_{g}(w)=w^{2-2 g} e_{g}\left(-w^{\nu-1} s\right)
$$

Using Lemma 4.3 we have

$$
\begin{array}{r}
\log \left(\frac{Z_{k+1}\left(-s_{k}\left(1+\frac{1}{k}\right)^{\nu-1}\right)}{Z_{k+1}(0)}\right)-2 \log \left(\frac{Z_{k}\left(-s_{k}\right)}{Z_{k}(0)}\right)+\log \left(\frac{Z_{k-1}\left(-s_{k}\left(1-\frac{1}{k}\right)^{\nu-1}\right)}{Z_{k-1}(0)}\right)= \\
=\left.\sum_{g \geq 0} \sum_{n \geq 1} k^{2-2 g-2 n} \frac{2}{(2 n) !} \frac{\partial^{(2 n)}}{\partial w^{(2 n)}}\left[w^{2-2 n} e_{g}\left(-w^{\nu-1} s_{k}\right)\right]\right|_{w=1} .
\end{array}
$$

In terms of the coefficients $z_{g}$ defined in Proposition 4.1 the right hand side of (4.39) becomes

$$
\log \left(\sum_{g \geq 0} k^{-2 g} z_{g}(s)\right) \text {. }
$$


We get ODE's which determine $e_{g}(-s)$ by equating like orders in equation (4.39) after making the substitutions (4.44) and (4.45). The equations so derived are

$$
\left.\sum_{n=0}^{g} \frac{2}{(2 n+2) !} \frac{\partial^{(2 n+2)}}{\partial w^{(2 n+2)}}\left[w^{2-2(g-n)} e_{g-n}\left(-w^{\nu-1} s\right)\right]\right|_{w=1}=\text { the } k^{-2 g} \text { term in } \log \left(\sum_{n=0} k^{-2 n} z_{n}(s)\right) .
$$

which establishes the hierarchy (2.9). These equations are recursive in the sense that we need to know $z_{n}$ for $n \leq g$ and $e_{m}$ for $m<g$ in order to write a closed differential equation for $e_{g}$. We will see how this works out in the next section.

\section{Continuum Toda Solutions and Fine Structure of the Asymptotic Partition Function}

In this section we will prove Theorems 2.3 and 2.5 The equations of the continuum Toda Lattice hierarchy are given by (2.7) and (2.8) and $b_{k}(t)$ possesses an asymptotic expansion whose leading order, $z_{0}(s)$, is the branch of the solution of the polynomial equation

$$
1=z_{0}(s)-c_{\nu} s z_{0}(s)^{\nu}
$$

which is regular at $s=0$. Recall that

$$
c_{\nu}=2 \nu\left(\begin{array}{c}
2 \nu-1 \\
\nu-1
\end{array}\right)
$$

Implicitly differentiating (5.1) we have the basic identity

$$
d s=\frac{\left(\nu-(\nu-1) z_{0}\right)}{c_{\nu} z_{0}^{\nu+1}} d z_{0} .
$$

which will be used often in following sections.

5.1. Integrating the Continuum Toda Lattice Hierarchy. Let $f_{g}(s, w)=w^{1-2 g} z_{g}\left(w^{\nu-1} s\right)$. The $k^{-2 g_{-}}$ order equation in (2.7) is linear in $z_{g}(s)$ :

$$
z_{g}^{\prime}(s)=\left.c_{\nu}\left(f_{0}^{\nu} f_{g w}+\nu f_{0}^{\nu-1} f_{g} f_{0 w}\right)\right|_{w=1}+\text { Forcing }\left._{g}\right|_{w=1}
$$

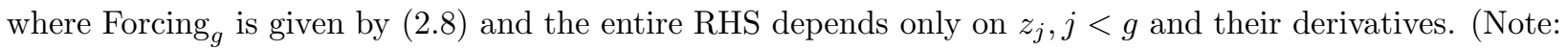
in what follows, if the context makes it clear that the forcing terms are being evaluated at $w=1$ we will simply write Forcing $g$ rather than Forcing $\left.\right|_{w=1}$.)

Expanding out the derivatives in equation (5.3),

$$
z_{g}^{\prime}=c_{\nu}\left((1-2 g) z_{0}^{\nu} z_{g}+(\nu-1) s z_{0}^{\nu} z_{g}^{\prime}+\nu z_{0}^{\nu} z_{g}+\nu(\nu-1) s z_{0}^{\nu-1} z_{g} z_{0}^{\prime}\right)+\text { Forcing }_{g} .
$$

Solving for $z_{g}^{\prime}$ :

$$
\left(1-c_{\nu}(\nu-1) s z_{0}^{\nu}\right) z_{g}^{\prime}=c_{\nu}\left((\nu+1-2 g) z_{0}^{\nu}+\nu(\nu-1) s z_{0}^{\nu-1} z_{0}^{\prime}\right) z_{g}+\text { Forcing }_{g}
$$

We may inductively assume that $z_{j}(s)$ is a function of $z_{0}(s)$ for $j<g$, and as a result Forcing $g$ is a function of $z_{0}$. One can convert equation (5.5) to a differential equation for $z_{g}$ as a function of $z_{0}$ using equations (5.11) and (5.2):

$$
\frac{d z_{g}}{d z_{0}}=\frac{\left(\nu(2-2 g)+(\nu-1)(2 g-1) z_{0}\right)}{z_{0}\left(\nu-(\nu-1) z_{0}\right)} z_{g}+\frac{\text { Forcing }_{g}}{c_{\nu} z_{0}^{\nu+1}} .
$$

Equation (5.6) is linear in $z_{g}$. Let

$$
\begin{aligned}
G\left(z_{0}, y\right) & =\int_{y}^{z_{0}} \frac{(\nu(2-2 g)+(\nu-1)(2 g-1) u)}{u(\nu-(\nu-1) u)} d u \\
& =\int_{y}^{z_{0}}\left((\nu-1)^{-1}(\nu-(\nu-1) u)^{-1}+2(1-g) u^{-1}\right) d u \\
& =-\log (\nu-(\nu-1) u)+\left.2(1-g) \log (u)\right|_{u=y} ^{u=z_{0}}
\end{aligned}
$$


Integrating equation (5.4) one has

$$
\begin{aligned}
z_{g}(s) & =\int_{1}^{z_{0}} \exp \left(G\left(z_{0}, y\right)\right) \frac{1}{c_{\nu} y^{\nu+1}} \text { Forcing }_{g}(y) d y \\
& =\frac{z_{0}^{2(1-g)}}{\nu-(\nu-1) z_{0}} \int_{1}^{z_{0}} \frac{\nu-(\nu-1) y}{c_{\nu} y^{\nu+3-2 g}} \operatorname{Forcing}_{g}(y) d y,
\end{aligned}
$$

where $z_{0}=z_{0}(s)$. This establishes the basic formula stated in Theorem 2.3 (3).

5.2. Singularities of $z_{g}$. In this section we complete the proof of Theorem 2.3 Expression (5.9) can be exploited to determine the singularity structure of $z_{g}(s)$. We differentiate equation (5.1) and solve for $z_{0}^{\prime}(s)$ :

$$
z_{0}^{\prime}(s)=\frac{c_{\nu} z_{0}^{\nu+1}}{\left(\nu-(\nu-1) z_{0}\right)}
$$

With equation (5.10) we can re-express successive derivatives of $z_{0}$ as functions of just $z_{0}$ by using the chain rule.

Doing so, we find the following result:

Lemma 5.1. The derivatives of $z_{0}(s)$ have the following form

$$
z_{0}^{(j)}(s)=\frac{c_{\nu}^{j} z_{0}^{\nu j+1}}{\left(\nu-(\nu-1) z_{0}\right)^{2 j-1}} \cdot(\text { Polynomial of degree } j-1)
$$

Equation (5.11) together with 5.2) give the following result

$$
s^{j} z_{0}^{(j)}(s)=\frac{z_{0}\left(z_{0}-1\right)^{j}}{\left(\nu-(\nu-1) z_{0}\right)^{2 j-1}} \cdot(\text { Polynomial of degree } j-1) .
$$

The terms in Forcing $(2.8$ ) are each of the form

$$
\left.\left(f_{k_{1}}\right)_{w^{\left(n_{1}\right)}}\left(f_{k_{2}}\right)_{w^{\left(n_{2}\right)}} \ldots\left(f_{k_{\nu+1}}\right)_{w^{\left(n_{\nu+1}\right)}}\right|_{w=1}
$$

where $f_{k}=w^{1-2 k} z_{k}\left(w^{\nu-1} s\right)$, and $n_{1}+n_{2}+\cdots+n_{\nu+1}=2\left(g-k_{1}-k_{2}-\cdots-k_{\nu+1}\right)$. Expanding the derivatives in (5.13) one finds that Forcing $g_{g}$ is in fact comprised of terms of the form:

$$
s^{j} z_{k_{1}}^{\left(j_{1}\right)} z_{k_{2}}^{\left(j_{2}\right)} \ldots z_{k_{\nu+1}}^{\left(j_{\nu+1}\right)}
$$

where

$$
\begin{gathered}
j=j_{1}+j_{2}+\cdots+j_{\nu+1}, \\
0 \leq j \leq 2\left(g-k_{1}-k_{2}-\cdots-k_{\nu+1}\right) .
\end{gathered}
$$

Lemma 5.2. The function $z_{g}^{(j)}(s)$ (j derivatives of $z_{g}(s)$ ) is of class iir with singularities occurring only at $z_{0}=0$ or $z_{0}=\nu /(\nu-1)$.

We will first prove by induction that $z_{g}(s)$ satisfies Lemma 5.2 see section 5.3 for the initial step. Assume that $z_{k}^{(j)}(s)$ for $k<g$ satisfies Lemma [5.2 this assumption, Lemma 5.1] and formula (5.1) show that the integrand in formula (5.9) is a polynomial in functions of class iir with singularities restricted to 0 and $\nu /(\nu-1)$ and having rational coefficients with at worst poles located at $z_{0}=0$. Thus the integral remains of class iir with the stated singularities and (5.9) is then this integral multiplied by a rational function with pole only at $\nu /(\nu-1)$.

We next prove, by induction on $j$, that the higher derivatives, $z_{g}^{(j)}(s)$, satisfy Lemma 5.2 which will complete the original induction. A derivative with respect to $z_{0}$ will raise the order of a singularity but will not introduce new singularities. Compute

$$
z_{g}^{(j)}(s)=\frac{d}{d z_{0}}\left[z_{g}^{(j-1)}(s)\right] z_{0}^{\prime}(s)
$$

by the induction step (in $j$ ) and formula (5.11) this expression is of class iir with singularities only at $z_{0}=0$ and $z_{0}=\nu /(\nu-1)$. 
With the forthcoming verification of the initial step of the induction (subsection 5.3), this completes the proof of Theorem 2.3

5.3. Example: $g=1$. Recall that $f_{0}(s, w)=w z_{0}\left(w^{\nu-1} s\right)$ and $f_{1}(s, w)=w^{-1} z_{1}\left(w^{\nu-1} s\right)$. The forcing for $z_{1}$ is given exclusively in terms of $z_{0}$ as

$$
c_{\nu}^{-1} \text { Forcing }_{1}=c_{\nu}^{-1} F_{1}^{\nu}[0]=\frac{\nu}{6} f_{0}^{\nu} f_{0 w w w}+\frac{\nu(\nu-1)}{3} f_{0}^{\nu-1} f_{0 w} f_{0 w w}+\frac{\nu(\nu-1)(\nu-2)}{12} f_{0}^{\nu-2} f_{0 w}^{3} .
$$

The entire expression is evaluated at $w=1$. We find that

$$
\begin{aligned}
\left.c_{\nu}^{-1} F_{1}^{\nu}[0]\right|_{w=1}= & \frac{\nu(\nu-1)(\nu-2)}{12} z_{0}^{\nu+1}+\frac{\nu(\nu-1)}{12}\left(9 \nu^{2}-17 \nu+6\right) s z_{0}^{\nu} z_{0}^{\prime}+\frac{5 \nu(\nu-1)^{3}}{6} s^{2} z_{0}^{\nu} z_{0}^{\prime \prime} \\
& +\frac{\nu(\nu-1)^{3}}{12}(7 \nu-6) s^{2} z_{0}^{\nu-1}\left(z_{0}^{\prime}\right)^{2}+\frac{\nu(\nu-1)^{3}}{6} s^{3} z_{0}^{\nu} z_{0}^{\prime \prime \prime}+\frac{\nu(\nu-1)^{4}}{3} s^{3} z_{0}^{\nu-1} z_{0}^{\prime} z_{0}^{\prime \prime} \\
& +\frac{\nu(\nu-1)^{4}(\nu-2)}{12} s^{3} z_{0}^{\nu-2}\left(z_{0}^{\prime}\right)^{3} .
\end{aligned}
$$

Inserting (5.16) into equation (5.9) with $g=1$, (writing all derivatives out as rational expressions of $z_{0}$ ) yields

$$
z_{1}(t)=\frac{(\nu-1) \nu\left(z_{0}-1\right) z_{0}\left(-\nu^{2}-2 z_{0}+\nu z_{0}+\nu^{2} z_{0}\right)}{12\left(\nu-(\nu-1) z_{0}\right)^{4}} .
$$

¿From this we see that not only does $z_{1}(t)$ satisfy Lemma 5.2 but in fact this expression is a rational function, without any of the potential complexities of the iir class. In fact all the examples we have worked out here for low values of $g$ yield rational expressions in $z_{0}$ for $z_{g}$. Moreover, the poles of these expressions are restricted to $z_{0}=\nu /(\nu-1)$.

Remark The rationality of (5.17) seems to depend very sensitively on the precise coefficients appearing

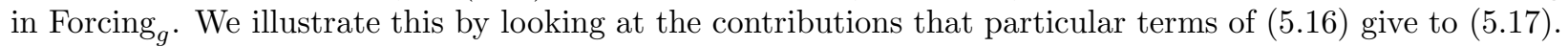
The integrand in integral (5.9) has a term of the form

$$
\frac{\left(\nu-(\nu-1) z_{0}\right)}{z_{0}^{\nu+1}} s^{2} z_{0}^{\nu-1}\left(z_{0}^{\prime}\right)^{2}=\frac{\left(z_{0}-1\right)^{2}}{\left(\nu-(\nu-1) z_{0}\right)},
$$

where we have used equation (5.10) to eliminate $z_{0}^{\prime}$. This term produces a logarithmic singularity at $\nu /(\nu-1)$; therefore one of the other terms must cancel this one to eliminate the logarithm.

¿From the representation (5.17) we can compute the Taylor Coefficients of $z_{1}(t)$ using the technique outlined in formulas (3.44 3.46):

$$
\begin{aligned}
\zeta_{j}^{(1)} & =\frac{c_{\nu}^{j}}{2 \pi i} \oint_{u \sim 1} \frac{(\nu-(\nu-1) u) u^{\nu j-1}}{(u-1)^{j+1}} z_{1}\left(z_{0}=u\right) d u \\
& =\frac{c_{\nu}^{j}}{2 \pi i} \oint_{u \sim 1} \frac{(\nu-1) \nu u^{\nu j}\left(-\nu^{2}-2 u+\nu u+\nu^{2} u\right)}{12(u-1)^{j}(\nu-(\nu-1) u)^{3}} d u \\
& =\frac{c_{\nu}^{j}}{2 \pi i} \oint_{w \sim 0} \frac{(\nu-1) \nu(w+1)^{\nu j}\left(\nu-2+\left(\nu^{2}+\nu-2\right) w\right)}{12(1-(\nu-1) w)^{3} w^{j}} d w .
\end{aligned}
$$

In the last line we have made the change of variables $w=u-1$. With the integral representation (5.18) one may conclude that

$$
\zeta_{j}^{(1)}=\nu c_{\nu}^{j}\left[w^{j-1} \text { Taylor Coefficient of } \frac{((\nu-1)(\nu-2)+(\nu+2) w)(w+1)^{\nu j}}{(1-(\nu-1) w)^{3}}\right] .
$$


5.4. Example: $g=2$. The forcing terms for $z_{2}(2.8)$ are:

(1) The first term is the summation in (2.8):

$$
c_{\nu} \nu f_{0}^{\nu-1} f_{1} f_{1_{w}}+c_{\nu} \nu(\nu-1) / 2 f_{0}^{\nu-2} f_{0 w} f_{1}^{2} .
$$

(2) The middle term is $F_{1}^{(\nu)}[2]$ :

$$
\begin{aligned}
c_{\nu}^{-1} F_{1}^{(\nu)}[2]= & \frac{\nu^{2}}{6} f_{0}^{\nu-1} f_{1} f_{0 w w w}+\frac{\nu}{6} f_{0}^{\nu} f_{1 w w w}+\frac{\nu(\nu-1)^{2}}{3} f_{0}^{\nu-2} f_{1} f_{0 w} f_{0 w w}+\frac{\nu(\nu-1)}{3} f_{0}^{\nu-1} f_{1 w} f_{0 w w} \\
& +\frac{\nu(\nu-1)}{3} f_{0}^{\nu-1} f_{0 w} f_{1 w w}+\frac{\nu(\nu-1)(\nu-2)^{2}}{12} f_{0}^{\nu-3} f_{1} f_{0 w}^{3}+\frac{\nu(\nu-1)(\nu-2)}{4} f_{0}^{\nu-2} f_{0 w}^{2} f_{1 w} .
\end{aligned}
$$

(3) The last term arises from $F_{2}^{(\nu)}[0]$ :

$$
\begin{aligned}
\left.\frac{1}{c_{\nu}} F_{2}^{(\nu)}[0]\right|_{w=1}= & \frac{\nu(2 \nu-1)}{120} f_{0}^{\nu} f_{0_{w}^{(5)}}+\frac{\nu(\nu-1)(18 \nu-11)}{360} f_{0}^{\nu-1} f_{0 w} f_{0 w^{(4)}}+\frac{\nu(\nu-1)(6 \nu-5)}{72} f_{0}^{\nu-1} f_{0 w w} f_{0 w w w} \\
& +\frac{\nu(\nu-1)(\nu-2)(23 \nu-20)}{360} f_{0}^{\nu-2} f_{0 w}^{2} f_{0 w w w}+\frac{\nu(\nu-1)(\nu-2)(62 \nu-65)}{720} f_{0}^{\nu-2} f_{0 w} f_{0 w w}^{2} \\
& +\frac{\nu(\nu-1)(\nu-2)(\nu-3)(16 \nu-19)}{360} f_{0}^{\nu-3} f_{0 w w} f_{0 w}^{3} \\
& +\frac{\nu(\nu-1)(\nu-2)(\nu-3)(\nu-4)(5 \nu-7)}{1440} f_{0}^{\nu-4} f_{0 w}^{5} .
\end{aligned}
$$

The three terms make up the forcing terms for $z_{2}$; each of them is expressed as a function of $z_{0}$. Substituting this explicit representation into equation (5.9) with $g=2$ we find that

$$
\begin{aligned}
z_{2}(t)= & \frac{1}{1440}(\nu-1) \nu\left(z_{0}-1\right) z_{0}\left[\left(2 \nu^{6}-14 \nu^{7}+24 \nu^{8}\right)\right. \\
& +\left(-12 \nu^{3}+148 \nu^{4}-546 \nu^{5}+758 \nu^{6}-252 \nu^{7}-96 \nu^{8}\right) z_{0} \\
& +\left(264 \nu^{2}-1510 \nu^{3}+25551 \nu^{4}-500 \nu^{5}-1789 \nu^{6}+840 \nu^{7}+144 \nu^{8}\right) z_{0}^{2} \\
& +\left(-536 \nu+1396 \nu^{2}+912 \nu^{3}-4596 \nu^{4}+2492 \nu^{5}+1296 \nu^{6}-868 \nu^{7}-96 \nu^{8}\right) z_{0}^{3} \\
& \left.+\left(168+234 \nu-1467 \nu^{2}+558 \nu^{3}+1902 \nu^{4}-1446 \nu^{5}-267 \nu^{6}+294 \nu^{7}+24 \nu^{8}\right) z_{0}^{4}\right] \\
& \cdot\left(\nu-(\nu-1) z_{0}\right)^{-9} .
\end{aligned}
$$

Let

$$
\begin{aligned}
r_{2}(w)= & \frac{c_{\nu}^{j} \nu(\nu-1)}{1440}(w+1)^{\nu j}[(\nu-2)(\nu-3)(\nu-4)(5 \nu-7) \\
& +2(\nu-1)(\nu-2)\left(168+84 \nu-246 \nu^{2}+73 \nu^{3}\right) w \\
& +(\nu-1)^{2}(\nu-2)\left(-504-1158 \nu+288 \nu^{2}+497 \nu^{3}\right) w^{2} \\
& +4(\nu-1)^{3}\left(-168-604 \nu-190 \nu^{2}+288 \nu^{3}+77 \nu^{4}\right) w^{3} \\
& \left.+3(\nu-1)^{4}\left(56+302 \nu+383 \nu^{2}+130 \nu^{3}+8 \nu^{4}\right) w^{4}\right](1-(\nu-1) w)^{-8} .
\end{aligned}
$$

We compute the Taylor Coefficients of $z_{2}(t)$ with a loop integral:

$$
\begin{aligned}
\zeta_{j}^{(2)} & =\frac{c_{\nu}^{j}}{2 \pi i} \oint_{u \sim 1} \frac{(\nu-(\nu-1) u) u^{\nu j-1}}{(u-1)^{j+1}} z_{2}\left(z_{0}=u\right) d u \\
& =\text { The } w^{j-1} \text { coefficient of } r_{2}(w) .
\end{aligned}
$$

5.5. Example: $g=3$. To compute $z_{3}$ we compute the forcing as above. Here we outline how the terms contributing to this forcing are found and then present the resulting explicit expression for $z_{3}$. There are six terms in formula (2.8) when $g=3$ : 
(1-2) The first two terms occur in the summation in (2.8) when $g=3$. One term contains three $f_{1}$ 's and is

$$
\frac{\nu(\nu-1)(\nu-2)}{6} f_{0}^{\nu-3} f_{1}^{3} f_{0 w}+\frac{\nu(\nu-1)}{2} f_{0}^{\nu-2} f_{1}^{2} f_{1 w}
$$

The other, containing an $f_{1}$ and an $f_{2}$, is

$$
\nu(\nu-1) f_{0}{ }^{\nu-2} f_{1} f_{2} f_{0 w}+\nu f_{0}^{\nu-1} f_{1} f_{2 w}+\nu f_{0}^{\nu-1} f_{2} f_{1 w} .
$$

(3-4) The next two terms are contributed by $F_{1}^{(\nu)}[4]$ : this expression represents the fourth order contributions of $F_{1}^{(\nu)}$. The terms involving two $f_{1}$ 's are

$$
\begin{aligned}
& \frac{\nu^{2}}{6} f_{0}^{\nu-1} f_{1} f_{1 w w w}+\frac{\nu^{2}(\nu-1)}{12} f_{0}^{\nu-2} f_{1}^{2} f_{0 w w w}+\frac{\nu(\nu-1)}{3} f_{0}^{\nu-1} f_{1 w} f_{1 w w}+\frac{\nu(\nu-1)^{2}}{3} f_{0}^{\nu-2} f_{1} f_{0 w} f_{1 w w} \\
& +\frac{\nu(\nu-1)^{2}}{3} f_{0}^{\nu-2} f_{1} f_{1 w} f_{0 w w}+\frac{\nu(\nu-1)^{2}(\nu-2)}{6} f_{0}^{\nu-3} f_{1}^{2} f_{0 w} f_{0 w w}+\frac{\nu(\nu-1)(\nu-2)}{4} f_{0}^{\nu-2} f_{0 w} f_{1 w}^{2} \\
& +\frac{\nu(\nu-1)(\nu-2)^{2}}{4} f_{0}^{\nu-3} f_{1} f_{0 w}^{2} f_{1 w}+\frac{\nu(\nu-1)(\nu-2)^{2}(\nu-3)}{24} f_{0}^{\nu-4} f_{1}^{2} f_{0 w}^{3} .
\end{aligned}
$$

The terms containing one $f_{2}$ are

$$
\begin{aligned}
& \frac{\nu}{6} f_{0}^{\nu} f_{2 w w w}+\frac{\nu^{2}}{6} f_{0}^{\nu-1} f_{2} f_{0 w w w}+\frac{\nu(\nu-1)}{3} f_{0}^{\nu-1} f_{0 w} f_{2 w w}+\frac{\nu(\nu-1)}{3} f_{0}^{\nu-1} f_{2 w} f_{0 w w} \\
& +\frac{\nu(\nu-1)^{2}}{3} f_{0}^{\nu-2} f_{2} f_{0 w} f_{0 w w}+\frac{\nu(\nu-1)(\nu-2)}{4} f_{0}^{\nu-2} f_{0 w}^{2} f_{2 w}+\frac{\nu(\nu-1)(\nu-2)^{2}}{12} f_{0}^{\nu-3} f_{2} f_{0 w}^{3}
\end{aligned}
$$

(5) The fifth term is

$$
\begin{aligned}
F_{2}^{(\nu)}[2]= & \frac{\nu(2 \nu-1)}{120} f_{0}^{\nu} f_{1 w^{(5)}}+\frac{\nu^{2}(2 \nu-1)}{120} f_{0}^{\nu-1} f_{1} f_{0 w}(5)+\frac{\nu(\nu-1)(18 \nu-11)}{360} f_{0}^{\nu-1} f_{0 w} f_{1 w^{(4)}} \\
& +\frac{\nu(\nu-1)(18 \nu-11)}{360} f_{0}^{\nu-1} f_{1 w} f_{0 w^{(4)}}+\frac{\nu(\nu-1)^{2}(18 \nu-11)}{360} f_{0}^{\nu-2} f_{1} f_{0 w} f_{0 w}(4) \\
& +\frac{\nu(\nu-1)(6 \nu-5)}{72} f_{0}^{\nu-1} f_{0 w w} f_{1 w w w}+\frac{\nu(\nu-1)(6 \nu-5)}{72} f_{0}^{\nu-1} f_{1 w w} f_{0 w w w} \\
& +\frac{\nu(\nu-1)^{2}(6 \nu-5)}{72} f_{0}^{\nu-2} f_{1} f_{0 w w} f_{0 w w w}+\frac{\nu(\nu-1)(\nu-2)(23 \nu-20)}{360} f_{0}^{\nu-2} f_{0 w}^{2} f_{1 w w w} \\
& +\frac{\nu(\nu-1)(\nu-2)(23 \nu-20)}{180} f_{0}^{\nu-2} f_{0 w} f_{1 w} f_{0 w w w}+\frac{\nu(\nu-1)(\nu-2)^{2}(23 \nu-20)}{360} f_{0}^{\nu-3} f_{1} f_{0 w}^{2} f_{0 w w w} \\
& +\frac{\nu(\nu-1)(\nu-2)(62 \nu-65)}{360} f_{0}^{\nu-2} f_{0 w} f_{0 w w} f_{1 w w}+\frac{\nu(\nu-1)(\nu-2)(62 \nu-65)}{720} f_{0}^{\nu-2} f_{1 w} f_{0 w w}^{2} \\
& +\frac{\nu(\nu-1)(\nu-2)^{2}(62 \nu-65)}{720} f_{0}^{\nu-3} f_{1} f_{0 w} f_{0 w w}^{2}+\frac{\nu(\nu-1)(\nu-2)(\nu-3)(16 \nu-19)}{360} f_{0}^{\nu-3} f_{0 w}^{3} f_{1 w w} \\
& +\frac{\nu(\nu-1)(\nu-2)(\nu-3)(16 \nu-19)}{120} f_{0}^{\nu-3} f_{0 w}^{2} f_{1 w} f_{0 w w} \\
& +\frac{\nu(\nu-1)(\nu-2)(\nu-3)^{2}(16 \nu-19)}{360} f_{0}^{\nu-4} f_{1} f_{0 w}^{3} f_{0 w w} \\
& +\frac{\nu(\nu-1)(\nu-2)(\nu-4)(5 \nu-7)}{288} f_{0}^{\nu-4} f_{0 w}^{4} f_{1 w}+\frac{\nu(\nu-1)(\nu-2)(\nu-4)^{2}(5 \nu-7)}{1440} f_{0}^{\nu-5} f_{1} f_{0 w}^{5} .
\end{aligned}
$$


(6) The last term is

$$
\begin{aligned}
F_{3}^{(\nu)}[0]= & \frac{\nu}{7 !}\left(3-8 \nu+6 \nu^{2}\right) f_{0}^{\nu} f_{0 w^{(7)}}+\frac{\nu(\nu-1)}{7 ! 3}\left(47-108 \nu+72 \nu^{2}\right) f_{0}^{\nu-1} f_{0 w} f_{0 w^{(6)}} \\
& +\frac{\nu(\nu-1)}{7 ! 2}\left(105-210 \nu+112 \nu^{2}\right) f_{0}^{\nu-1} f_{0 w w} f_{0 w^{(5)}}+\frac{\nu(\nu-1)}{7 ! 6}\left(539-1050 \nu+504 \nu^{2}\right) f_{0}^{\nu-1} f_{0 w^{(3)}} f_{0 w^{(4)}} \\
& +\frac{\nu(\nu-1)(\nu-2)}{7 ! 2}\left(86-163 \nu+86 \nu^{2}\right) f_{0}^{\nu-2} f_{0 w}^{2} f_{0 w^{(5)}} \\
& +\frac{\nu(\nu-1)(\nu-2)}{7 ! 6}\left(1387-2416 \nu+1044 \nu^{2}\right) f_{0}^{\nu-2} f_{0 w} f_{0 w w} f_{0 w^{(4)}} \\
& +\frac{\nu(\nu-1)(\nu-2)}{7 ! 3}\left(467-803 \nu+327 \nu^{2}\right) f_{0}^{\nu-2} f_{0 w} f_{0 w^{(3)}}^{2} \\
& +\frac{\nu(\nu-1)(\nu-2)}{7 ! 6}\left(1456-2359 \nu+882 \nu^{2}\right) f_{0}^{\nu-2} f_{0 w w}^{2} f_{0 w^{(3)}} \\
& +\frac{\nu(\nu-1)(\nu-2)(\nu-3)}{7 ! 6}\left(410-665 \nu+270 \nu^{2}\right) f_{0}^{\nu-3} f_{0 w}^{3} f_{0 w^{(4)}} \\
& +\frac{\nu(\nu-1)(\nu-2)(\nu-3)}{7 ! 2}\left(857-1309 \nu+458 \nu^{2}\right) f_{0}^{\nu-3} f_{0 w}^{2} f_{0 w w} f_{0 w^{(3)}} \\
& +\frac{\nu(\nu-1)(\nu-2)(\nu-3)}{7 ! 6}\left(1327-1932 \nu+620 \nu^{2}\right) f_{0}^{\nu-3} f_{0 w} f_{0 w w}^{3} \\
& +\frac{\nu(\nu-1)(\nu-2)(\nu-3)(\nu-4)}{7 ! 12}\left(788-1121 \nu+359 \nu^{2}\right) f_{0}^{\nu-4} f_{0 w}^{4} f_{0 w^{(3)}} \\
& +\frac{\nu(\nu-1)(\nu-2)(\nu-3)(\nu-4)}{7 ! 12}\left(2431-3315 \nu+974 \nu^{2}\right) f_{0}^{\nu-4} f_{0 w}^{3} f_{0 w w}^{2} \\
& +\frac{\nu(\nu-1)(\nu-2)(\nu-3)(\nu-4)(\nu-5)}{7 ! 6}\left(228-290 \nu+77 \nu^{2}\right) f_{0}^{\nu-5} f_{0 w}^{5} f_{0 w w} \\
& +\frac{\nu(\nu-1)(\nu-2)(\nu-3)(\nu-4)(\nu-5)(\nu-6)}{7 ! 72}\left(124-147 \nu+35 \nu^{2}\right) f_{0}^{\nu-6} f_{0 w}^{7} .
\end{aligned}
$$

The entire forcing expression is a rational function of $z_{0}$ with singularities only at $z_{0}=\nu /(\nu-1)$. We insert these forcing terms into equation (5.9) with $g=3$ and find that:

$$
\begin{aligned}
& z_{3}(t)=\frac{\nu(\nu-1)}{362880} \frac{z_{0}\left(z_{0}-1\right)}{\left(\nu-(\nu-1) z_{0}\right)^{14}} \\
& {\left[(\nu-2)(\nu-3)(\nu-4)(\nu-5)(\nu-6)\left(124-147 \nu+35 \nu^{2}\right)\right.} \\
& +(\nu-3)(\nu-2)(\nu-1)\left(104160+47584 \nu-332550 \nu^{2}+270697 \nu^{3}-83226 \nu^{4}+8923 \nu^{5}\right)\left(z_{0}-1\right) \\
& +3(\nu-2)(\nu-1)^{2}\left(312480+744980 \nu-1245750 \nu^{2}+373091 \nu^{3}+1085920 \nu^{4}-485414 \nu^{5}\right. \\
& \left.+67225 \nu^{6}\right)\left(z_{0}-1\right)^{2} \\
& +(\nu-2)(\nu-1)^{3}\left(-1562400-7251840 \nu+290690 \nu^{2}+11468057 \nu^{3}-2824078 \nu^{4}-3154302 \nu^{5}\right. \\
& \left.+1078663 \nu^{6}\right)\left(z_{0}-1\right)^{3} \\
& +(\nu-2)(\nu-1)^{4}\left(1562400+10781280 \nu+12588010 \nu^{2}-10677353 \nu^{3}-11255921 \nu^{4}+3006363 \nu^{5}\right. \\
& \left.+1779986 \nu^{6}\right)\left(z_{0}-1\right)^{4} \\
& +3(\nu-1)^{5}\left(624960+5411808 \nu+10100796 \nu^{2}-1315908 \nu^{3}-9371695 \nu^{4}-973573 \nu^{5}+1835799 \nu^{6}\right. \\
& \left.+308858 \nu^{7}\right)\left(z_{0}-1\right)^{5} \\
& +(\nu-1)^{6}\left(-624960-6823584 \nu-20098900 \nu^{2}-16851720 \nu^{3}+3867117 \nu^{4}+8356442 \nu^{5}\right. \\
& \left.+2223760 \nu^{6}+119824 \nu^{7}\right)\left(z_{0}-1\right)^{6} \\
& +5(\nu-1)^{7}\left(17856+235296 \nu+939236 \nu^{2}+1505064 \nu^{3}+1032603 \nu^{4}+285860 \nu^{5}+24472 \nu^{6}\right. \\
& \left.\left.+64 \nu^{7}\right)\left(z_{0}-1\right)^{7}\right] \text {. }
\end{aligned}
$$


Let

$$
\begin{aligned}
r_{3}(w)= & \frac{c_{\nu}^{j} \nu(\nu-1)}{362880}(w+1)^{\nu j}\left[(\nu-2)(\nu-3)(\nu-4)(\nu-5)(\nu-6)\left(124-147 \nu+35 \nu^{2}\right)\right. \\
+ & (\nu-3)(\nu-2)(\nu-1)\left(104160+47584 \nu-332550 \nu^{2}+270697 \nu^{3}-83226 \nu^{4}+8923 \nu^{5}\right) w \\
+ & 3(\nu-2)(\nu-1)^{2}\left(312480+744980 \nu-1245750 \nu^{2}+373091 \nu^{3}+1085920 \nu^{4}-485414 \nu^{5}+67225 \nu^{6}\right) w^{2} \\
+ & (\nu-2)(\nu-1)^{3}\left(-1562400-7251840 \nu+290690 \nu^{2}+11468057 \nu^{3}-2824078 \nu^{4}-3154302 \nu^{5}\right. \\
& \left.+1078663 \nu^{6}\right) w^{3} \\
+ & +(\nu-2)(\nu-1)^{4}\left(1562400+10781280 \nu+12588010 \nu^{2}-10677353 \nu^{3}-11255921 \nu^{4}+3006363 \nu^{5}\right. \\
& \left.+308858 \nu^{7}\right) w^{5} \\
+ & \left.\quad+2223760 \nu^{6}+119824 \nu^{7}\right) w^{6} \\
+ & (\nu-1)^{6}\left(-624960-6823584 \nu-20098900 \nu^{2}-16851720 \nu^{3}+3867117 \nu^{4}+8356442 \nu^{5}\right. \\
+ & \left.\left.+64 \nu^{7}\right) w^{7}\right](1-(\nu-1) w)^{-13} .
\end{aligned}
$$

We compute the Taylor Coefficients of $z_{3}(t)$ with a loop integral:

$$
\begin{aligned}
\zeta_{j}^{(3)} & =\frac{c_{\nu}^{j}}{2 \pi i} \oint_{u \sim 1} \frac{(\nu-(\nu-1) u) u^{\nu j-1}}{(u-1)^{j+1}} z_{3}\left(z_{0}=u\right) d u \\
& =\text { The } w^{j-1} \text { coefficient of } r_{3}(w) .
\end{aligned}
$$

5.6. Determining $e_{g}\left(-s_{2 \nu}\right)$. Theorem 2.4 gives a second order differential equation determining $e_{g}\left(-s_{2 \nu}\right)$ :

$$
\frac{\partial^{2}}{\partial w^{2}}\left[w^{2-2 g} e_{g}\left(-w^{\nu-1} s\right)\right]=\operatorname{drivers}_{g},
$$

where the entire equation is evaluated at $w=1$ and where the drivers are the right hand side of equation (2.9):

$$
-\sum_{n=1}^{g} \frac{2}{(2 n+2) !} \frac{\partial^{2 n+2}}{\partial w^{2 n+2}}\left[w^{2-2(g-n)} e_{g-n}\left(-w^{\nu-1} s\right)\right]+\text { the } k^{-2 g} \text { term of } \log \left[\sum_{n=0}^{\infty} \frac{1}{k^{2 n}} z_{n}(s)\right] .
$$

Expanding the two derivatives on the left hand side of (5.19) and setting $w=1$ we arrive at the basic ode for the $e_{g}$ :

$$
(2-2 g)(1-2 g) e_{g}(-s)-(\nu-1)(\nu+2-4 g) s e_{g}^{\prime}(-s)+(\nu-1)^{2} s^{2} e_{g}^{\prime \prime}(-s)=\text { drivers }_{g} .
$$

If $g \neq 1$ one solves this equation by integrating factors as follows: Multiply both sides of (5.20) by $s^{\gamma_{1}}$ with

$$
\gamma_{1}=\gamma_{1}^{(+)}=\frac{3-\nu-2 g}{\nu-1} \quad \text { or } \quad \gamma_{1}^{(-)}=\frac{2-\nu-2 g}{\nu-1}
$$

to arrive at

$$
(2-2 g)(1-2 g) s^{\gamma_{1}} e_{g}(-s)-(\nu-1)(\nu+2-4 g) s^{\gamma_{1}+1} e_{g}^{\prime}(-s)+(\nu-1)^{2} s^{\gamma_{1}+2} e_{g}^{\prime \prime}(-s)=s^{\gamma_{1}} \text { drivers }_{g} .
$$

Let

$$
A=(2-2 g)(1-2 g) /\left(\gamma_{1}+1\right)
$$

We then integrate equation (5.22) once,

$$
A s^{\gamma_{1}+1} e_{g}(-s)-(\nu-1)^{2} s^{\gamma_{1}+2} e_{g}^{\prime}(-s)=\int_{0}^{s} s_{1}{ }^{\gamma_{1}} \operatorname{drivers}_{g}\left(s_{1}\right) d s_{1}+K_{1}^{\prime},
$$


where $K_{1}^{\prime}$ is a constant of integration. Multiplying by $s^{\gamma_{2}}$,

$$
A s^{\gamma_{1}+\gamma_{2}+1} e_{g}(s)-(\nu-1)^{2} s^{\gamma_{1}+\gamma_{2}+2} e_{g}^{\prime}(-s)=s^{\gamma_{2}} \int_{0}^{s} s_{1}^{\gamma_{1}} \operatorname{drivers}_{g}\left(s_{1}\right) d s_{1}+s^{\gamma_{2}} K_{1}^{\prime},
$$

we see that if $\gamma_{2}$ satisfies

$$
\gamma_{2}=\frac{(2-2 g)(1-2 g)-(\nu-1)^{2}\left(\gamma_{1}+1\right)\left(\gamma_{1}+2\right)}{\left(\gamma_{1}+1\right)(\nu-1)^{2}},
$$

one may solve equation (5.24) for $e_{g}(s)$ :

$$
e_{g}(-s)=\frac{1}{(\nu-1)^{2}} s^{-\gamma_{1}-\gamma_{2}-2} \int_{0}^{s} s_{1}^{\gamma_{2}} \int_{0}^{s_{1}} s_{2}^{\gamma_{1}} \operatorname{drivers}_{g}\left(s_{2}\right) d s_{2} d s_{1}+K_{1} s^{-\gamma_{1}-1}+K_{2} s^{-\gamma_{1}-\gamma_{2}-2},
$$

where $K_{1}$ and $K_{2}$ are constants of integration.

Switching the order of integration in (5.26)

$$
e_{g}(-s)=\frac{1}{(\nu-1)^{2}} s^{-\gamma_{1}-\gamma_{2}-2} \int_{0}^{s} \int_{s_{2}}^{s} s_{1}^{\gamma_{2}} s_{2}^{\gamma_{1}} \operatorname{drivers}_{g}\left(s_{2}\right) d s_{1} d s_{2}+K_{1} s^{-\gamma_{1}-1}+K_{2} s^{-\gamma_{1}-\gamma_{2}-2},
$$

one can compute the $s_{1}$ integral in (5.27):

$$
\begin{aligned}
e_{g}(-s)= & \frac{1}{(\nu-1)^{2}} \frac{1}{\gamma_{2}+1}\left[s^{-\gamma_{1}-1} \int_{0}^{s} s_{2}^{\gamma_{1}} \operatorname{drivers}_{g} d s_{2}-s^{-\gamma_{1}-\gamma_{2}-2} \int_{0}^{s} s_{2}^{\gamma_{1}+\gamma_{2}+2} \operatorname{drivers}_{g} d s_{2}\right] \\
& +K_{1} s^{-\gamma_{1}-1}+K_{2} s^{-\gamma_{1}-\gamma_{2}-2} .
\end{aligned}
$$

Applying an integration by parts to the remaining integrals (integrating the power of $s_{2}$ and differentiating the drivers (with respect to $\left.s_{2}\right)$ ):

$$
\begin{aligned}
e_{g}(-s)= & \frac{1}{(\nu-1)^{2}} \frac{1}{\left(\gamma_{2}+1\right)}\left[\left(\frac{1}{\gamma_{1}+1}-\frac{1}{\gamma_{1}+\gamma_{2}+2}\right) \operatorname{drivers}_{g}+\frac{s^{-\gamma_{1}-1}}{\gamma_{1}+1} \int_{0}^{s} s_{2}^{\gamma_{1}+1}\left(\operatorname{drivers}_{g}\right) \bullet z_{0}^{\prime} d s_{2}\right. \\
& \left.-\frac{s^{-\gamma_{1}-\gamma_{2}-2}}{\gamma_{1}+\gamma_{2}+2} \int_{0}^{s} s_{2}^{\gamma_{1}+\gamma_{2}+2}\left(\operatorname{drivers}_{g}\right) z_{0}^{\prime} d s_{2}\right]+K_{1} s^{-\gamma_{1}-1}+K_{2} s^{-\gamma_{1}-\gamma_{2}-2} .
\end{aligned}
$$

We may now change variables and integrate with respect to $y=z_{0}\left(s_{2}\right)$ in (5.29):

$$
\begin{aligned}
e_{g}(-s)= & \frac{1}{(\nu-1)^{2}\left(\gamma_{2}+1\right)}\left[\frac{\gamma_{2}+1}{\left(\gamma_{1}+1\right)\left(\gamma_{1}+\gamma_{2}+2\right)} \operatorname{drivers}_{g}\right. \\
- & \frac{1}{\left(\gamma_{1}+1\right)}\left(\frac{c_{\nu} z_{0}^{\nu}}{z_{0}-1}\right)^{\gamma_{1}+1} \int_{1}^{z_{0}}\left(\frac{y-1}{c_{\nu} y^{\nu}}\right)^{\gamma_{1}+1}\left(\text { drivers }_{g}\right)^{\bullet} d y \\
& \left.+\frac{1}{\gamma_{1}+\gamma_{2}+2}\left(\frac{c_{\nu} z_{0}^{\nu}}{z_{0}-1}\right)^{\gamma_{1}+\gamma_{2}+2} \int_{1}^{z_{0}}\left(\frac{y-1}{c_{\nu} y^{\nu}}\right)^{\gamma_{1}+\gamma_{2}+2}\left(\text { drivers }_{g}\right)^{\bullet} d y\right] \\
& +K_{1} s^{-\gamma_{1}-1}+K_{2} s^{-\gamma_{1}-\gamma_{2}-2}
\end{aligned}
$$

where $K_{1}$ and $K_{2}$ are constants determined in either of the following ways: (1) by the requirement that $e_{g}$ be a locally analytic function of $s$ or (2) by the evaluation of $e_{g}$ for low values of $\nu$ through its combinatorial characterization.

Direct calculation shows that either choice of $\gamma_{1}$ in (5.21) produces formula (2.10) in Theorem 2.5

5.7. Example $g=0$. When $g=0$ we have

$$
\gamma_{1}=\gamma_{1}^{(-)}=-\frac{\nu-2}{\nu-1}
$$

then

$$
\gamma_{2}=-\frac{\nu-2}{\nu-1}
$$


The driver when $g=0$ is just $\log \left(z_{0}\right)$. The two integrals in (5.31) may be evaluated separately:

$$
\begin{aligned}
\int^{z_{0}}(y-1)^{1 /(\nu-1)} y^{-(2 \nu-1) /(\nu-1)} d y & =\int_{1}^{z_{0}}\left(1-y^{-1}\right)^{1 /(\nu-1)} y^{-2} d y \\
& =\frac{\nu-1}{\nu}\left(1-z_{0}^{-1}\right)^{\nu /(\nu-1)}
\end{aligned}
$$

and

$$
\begin{aligned}
\int^{z_{0}}(y-1)^{2 /(\nu-1)} y^{-(3 \nu-1) /(\nu-1)} d y= & \int_{1}^{z_{0}}\left(1-y^{-1}\right)^{2 /(\nu-1)} y^{-3} d y \\
= & \frac{(\nu-1)}{(\nu+1)}\left(1-z_{0}^{-1}\right)^{(\nu+1) /(\nu-1)} z_{0}^{-1} \\
& +\frac{(\nu-1)}{(\nu+1)} \int_{1}^{z_{0}}\left(1-y^{-1}\right)^{(\nu+1) /(\nu-1)} y^{-2} d y \\
= & \frac{(\nu-1)}{(\nu+1)}\left(\frac{z_{0}-1}{z_{0}^{\nu}}\right)^{(\nu+1) /(\nu-1)} z_{0}^{\nu}+\frac{(\nu-1)^{2}}{2 \nu(\nu+1)}\left(\frac{z_{0}-1}{z_{0}^{\nu}}\right)^{2 \nu /(\nu-1)} z_{0}^{2 \nu} .
\end{aligned}
$$

Plugging (5.32) and (5.33) into (5.31) with $g=0$ yields:

$$
\begin{aligned}
e_{0}(-s) & =\frac{1}{2} \log \left(z_{0}\right)-\frac{\nu-1}{\nu}\left(z_{0}-1\right)+\frac{1}{2} \frac{\nu-1}{\nu+1}\left(z_{0}-1\right)+\frac{1}{4} \frac{(\nu-1)^{2}}{4 \nu(\nu+1)}\left(z_{0}-1\right)^{2} \\
& =\frac{1}{2} \log \left(z_{0}\right)+\frac{(\nu-1)^{2}}{4 \nu(\nu+1)}\left(z_{0}-1\right)\left(z_{0}-\frac{3(\nu+1)}{\nu-1}\right)
\end{aligned}
$$

where $K_{1}$ and $K_{2}$ are both 0 by the analyticity condition. This formula for $e_{0}$ agrees with the expression for $e_{0}(s)$ found using the equilibrium measure.

5.8. Example: $g=1$. The drivers for $e_{1}$ are

$$
\frac{z_{1}}{z_{0}}-\frac{1}{12} \frac{\partial^{4}}{\partial w^{4}}\left(w^{2} e_{0}\left(-w^{\nu-1} s\right)\right)
$$

evaluated at $w=1$. As with the Forcing terms for the $z_{g}$ this formula can be expressed as a rational function of $z_{0}$ with poles at $z_{0}=0$ and $z_{0}=\nu /(\nu-1)$.

We find that $g=1$ is the exceptional case for the method used in formulas (5.22)-(5.31). We compute it directly from equation (5.20) with $g=1$. The integrating factors are $\left(\gamma_{1}, \gamma_{2}\right)=(-1,-\nu /(\nu-1))$, differing from the general choice of integrating factors (5.21) and (5.25).

We find the integral formula:

$$
\begin{aligned}
e_{1}(-s)= & \frac{1}{(\nu-1)^{2}} s^{1 /(\nu-1)} \int_{0}^{s} \int_{0}^{s} s_{1}^{-\nu /(\nu-1)} s_{2}^{-1} \operatorname{drivers}_{1}\left(s_{2}\right) d s_{2} d s_{1}+K_{1}+K_{2} s^{1 /(\nu-1)} \\
= & \frac{1}{(\nu-1)^{2}} s^{1 /(\nu-1)} \int_{0}^{s} \int_{s_{2}}^{s} s_{1}^{-\nu /(\nu-1)} s_{2}^{-1} \operatorname{drivers}_{1}\left(s_{2}\right) d s_{1} d s_{2}+K_{1}+K_{2} s^{1 /(\nu-1)} \\
= & \frac{1}{(\nu-1)}\left[s^{1 /(\nu-1)} \int_{0}^{s} s_{2}^{-\nu /(\nu-1)} \operatorname{drivers}_{1} d s_{2}-\int_{0}^{s} s_{2}^{-1} \operatorname{drivers}_{1} d s_{2}\right]+K_{1}+K_{2} s^{1 /(\nu-1)} \\
= & \frac{1}{(\nu-1)}\left[\left(\frac{z_{0}(s)-1}{c_{\nu} z_{0}(s)^{\nu}}\right)^{1 /(\nu-1)} \int_{1}^{z_{0}(s)}\left(\frac{c_{\nu} y^{\nu}}{y-1}\right)^{\nu /(\nu-1)} \frac{(\nu-(\nu-1) y)}{c_{\nu} y^{\nu+1}} \operatorname{drivers}_{1} d y\right. \\
& \left.\quad-\int_{1}^{z_{0}(s)} \frac{(\nu-(\nu-1) y)}{y(y-1)} \operatorname{drivers}_{1} d y\right]+K_{1}+K_{2} s^{1 /(\nu-1)} \\
= & -\frac{1}{12} \log \left(\nu+(1-\nu) z_{0}\right)+K_{1}+K_{2} s^{1 /(\nu-1)} ;
\end{aligned}
$$

where we have switched the order of integration, computed the $s_{1}$ integral, and changed coordinates to integrals with respect to $y=z_{0}\left(s_{2}\right)$. The final step is a direct computation and is left to the reader. 
When $t=0, \log \left(Z_{N}(t) / Z_{N}(0)\right)=0$, therefore $e_{1}(0)=0$ and so $K_{1}=0$. We notice that if $\nu \neq 2$ then $K_{2}=0$ by the analyticity condition, otherwise we will have to determine $K_{2}$. The computation we will carry out in Section [5.10] shows that when $\nu=2$,

$$
e_{1}(-s)=s+\mathcal{O}\left(s^{2}\right) \text {. }
$$

Our expression for $e_{1}(-s)$ has the expansion (when $\nu=2$ )

$$
-\frac{1}{12} \log \left[2-z_{0}(s)\right]+K_{2} s=\left(1+K_{2}\right) s+\mathcal{O}\left(s^{2}\right),
$$

therefore $K_{2}=0$. This proves formula 2.11).

5.9. Example: $g=2$. The drivers for $e_{2}(s)$ are

$$
\frac{z_{2}}{z_{0}}-\frac{1}{2} \frac{z_{1}^{2}}{z_{0}^{2}}-\frac{1}{12} \frac{\partial^{4}}{\partial w^{4}}\left(e_{1}\left(-w^{\nu-1} s\right)\right)-\frac{1}{360} \frac{\partial^{6}}{\partial w^{6}}\left(w^{2} e_{0}\left(-w^{\nu-1} s\right)\right),
$$

evaluated at $w=1$. As with the Forcing terms for the $z_{g}$ and the drivers for $e_{1}$ this expression can be expressed as a rational function of $z_{0}$ with poles at $z_{0}=0$ and $z_{0}=\nu /(\nu-1)$.

For $g=2$ we find that

and

$$
\gamma_{1}=\gamma_{1}^{(+)}=-\frac{\nu+1}{\nu-1}
$$

$$
\gamma_{2}=-\frac{\nu}{\nu-1}
$$

We compute the integrals in equation (5.31),

$$
\begin{aligned}
e_{2}(-s)= & \frac{1}{2880}(\nu-1)\left(z_{0}-1\right)\left(\nu-(\nu-1) z_{0}\right)^{-5}\left(\left(-\nu^{3}+5 \nu^{4}+8 \nu^{5}\right)\right. \\
& +\left(-\nu^{2}+41 \nu^{3}-24 \nu^{4}-16 \nu^{5}\right) z_{0}+\left(44 \nu-89 \nu^{2}+54 \nu^{3}-17 \nu^{4}+8 \nu^{5}\right) z_{0}^{2} \\
& \left.+\left(-12-12 \nu+108 \nu^{2}-132 \nu^{3}+48 \nu^{4}\right) z_{0}^{3}+\left(-12+48 \nu-72 \nu^{2}+48 \nu^{3}-12 \nu^{4}\right) z_{0}^{4}\right) \\
& +K_{1} s^{2 /(\nu-1)}+K_{2} s^{3 /(\nu-1)} .
\end{aligned}
$$

We notice that $K_{1}$ and $K_{2}$ will be zero by the analyticity condition unless $\nu=2,3$, or 4 in which case they will have to be determined by some other means such as that illustrated in the next subsection.

5.10. Evaluating the constants of integration. We will now outline a method for determining the constants of integration in formulas (2.10) and (2.11) of Theorem 2.5]

We know, from [15], that $e_{g}(-s)$ is analytic in $s$ in a neighborhood of $s=0$, therefore we notice that for many values of $\gamma_{1}$ both $K_{1}$ and $K_{2}$ will vanish to preserve analyticity. However there are some values for which they will not: Values of $\nu$ and $g$ which conspire to make $(2 g-2) /(\nu-1)$ or $(2 g-1) /(\nu-1)$ be positive integers. To find these constants, when necessary, one may rely on the combinatorial interpretation of the Taylor coefficients of $e_{g}$ described in Theorem [1.3. Here we outline the method that we have used to evaluate these constants for $g \leq 3$.

The set of maps $D$ having vertex set $K_{0}(D)$ of fixed cardinality at specified vertices can be placed in 1-1 correspondence with a class of subgroups of the permutation group $\mathcal{S}_{d}$ where $d$, twice the number of edges, is determined by the cardinality and valences of $K_{0}(D)$ 뜨. For simplicity and also because it is the case of relevance for us, we take $K_{0}(D)$ to consist of $n$ vertices each of valence $2 \nu$. For this class $d=2 \nu n$ which is the cardinality of the set of darts of $K(D)$. A dart is an oriented edge. Equivalently, we may define the abstract set of darts associated to this class of maps as

$$
\Omega=\Omega_{1} \cup \Omega_{2},
$$

where

$$
\Omega_{1}=\{(v, e): v \text { is a vertex and } e \text { is an edge with two distinct vertices one of them being } v\},
$$

and

$$
\Omega_{2}=\{(v, e, \pm): v \text { is a vertex and } e \text { is an edge with a single vertex } v\}
$$


The element $(v, e) \in \Omega_{1}$ represents the dart based at $v$ and going along $e$. The element $(v, e, \pm) \in \Omega_{2}$ represents the dart based at $v$ going along $e$ in the counterclockwise (resp. clockwise) orientation. For each edge there are two darts, therefore $|\Omega|=d$ and we can think of $\mathcal{S}_{d}$ as acting by permutations on the set $\Omega$.

Given a map, $(K(D),[\iota])$ with $K_{0}(D)$ specified as above, we define a subgroup of $\mathcal{S}_{d}$ generated by two permutations $\langle\sigma, \tau\rangle$. The orientation on $X$ induces (via $[\iota]$ ) a cyclic ordering on the darts attatched to each vertex; the first permutation $\sigma$ is given by this action. Explicitly, $\sigma$ maps the element $(v, e) \in \Omega_{1}$ to the element $(v, \tilde{e}) \in \Omega_{1}$ or $(v, \tilde{e}, \pm) \in \Omega_{2}$ where $\tilde{e}$ is the edge counter clockwise in the orientation at $v$ from $e$. Likewise $\sigma$ maps the element $(v, e, \pm) \in \Omega_{2}$ to the element $(v, \tilde{e}) \in \Omega_{1}$ or $(v, \tilde{e}, \pm) \in \Omega_{2}$ where $\tilde{e}$ is the edge counter clockwise in the orientation at $v$ from $e$. The second permutation $\tau$, is given explicitly as the permutation which acts on $\Omega_{1}$ by sending $(v, e)$ to $(\tilde{v}, e)$ where $\tilde{v}$ is the other endpoint of $e ; \tau$ acts on $\Omega_{2}$ by sending $(v, e, \pm)$ to $(v, e, \mp)$.

¿From these descriptions one sees that: $\sigma$ is a product of disjoint $2 \nu$-cycles, with each cycle corresponding to a unique vertex in $K_{0}(D)$; and that $\tau$ is a product of disjoint 2-cycles, with each 2-cycle corresponding to a unique edge in $K_{1}(D)$.

Conversely, given a subgroup presented as above which also has the property that the group acts transitively on $\Omega$, one may construct a unique map. The transitivity condition insures that the underlying map is connected. The permutation $\tau$ determines how the vertices are connected through edges to define the graph. The permutation $\sigma$ gives the orientation of the edges about each vertex. Together these two permutations determine the surface $X$ and the embedding class $[\iota]$.

The punchline is that what we now have is an algorithm for computing the coefficient of $s^{n} / n$ ! in $e_{g}(-s)$ for finite $g, \nu$, and $n$ : Let $d=2 \nu n$. Fix $\sigma$ to be a permutation formed by a disjoint product of $n 2 \nu$-cycles in $\mathcal{S}_{d}$. Then we choose each disjoint product of $\nu n$ 2-cycles, $\tau$ in $\mathcal{S}_{d}$. Check if $(\sigma, \tau)$ is connected (by verifying that the orbit of $\langle\sigma, \tau\rangle \cdot 1$ is all $d$ letters). If $(\sigma, \tau)$ is connected compute the genus by Euler's formula $(1-\nu) n+F=\chi=2-2 g$, where $F$ (the number of faces) is given by the number of cycles in $\sigma \circ \tau$. The details of this calculation together with some examples and a generalization to the case of unoriented maps are in a forthcoming paper [23].

The algorithm gives the following values for the coefficient of $s^{j} / j$ ! in $e_{g}(-s)$ :

\begin{tabular}{|lllr|}
\hline$g=1$ & & $j=0$ & 0 \\
$g=1$ & $\nu=2$ & $j=1$ & 1 \\
\hline$g=2$ & $\nu=2$ & $j=2$ & 0 \\
$g=2$ & $\nu=2$ & $j=3$ & 1440 \\
$g=2$ & $\nu=3$ & $j=1$ & 0 \\
$g=2$ & $\nu=4$ & $j=1$ & 21 \\
\hline$g=3$ & $\nu=2$ & $j=4$ & 0 \\
$g=3$ & $\nu=2$ & $j=5$ & 58060800 \\
$g=3$ & $\nu=3$ & $j=2$ & 0 \\
$g=3$ & $\nu=5$ & $j=1$ & 0 \\
$g=3$ & $\nu=6$ & $j=1$ & 1485 \\
\hline
\end{tabular}

Then $K_{1}$ and $K_{2}$ are chosen so that the $j$ 'th coefficient of $e_{g}(-s)$ matches these numbers.

5.11. Multiple times . The constructions carried out in this paper extend to multiple even time parameters; this is a reflection of the commutativity of the underlying flows in the Toda Lattice hierarchy. However, the expressions found are more complicated and are less easily reduced to closed form than the monic time cases we have considered thus far.

For brevity we will show how our results extend to two times: $t_{2 \nu_{1}}$ and $t_{2 \nu_{2}}$, where $\nu_{1}$ and $\nu_{2}$ are positive integers. Theorem 2.2 becomes

Theorem 5.3. In the limit as $k \rightarrow \infty, b_{k}\left(\xi_{2 \nu_{1}}, \xi_{2 \nu_{2}}\right)^{2}$ has a valid asymptotic expansion of the form

$$
b_{k}\left(\xi_{2 \nu_{1}}, \xi_{2 \nu_{2}}\right)^{2} \simeq k\left(z_{0}\left(s_{2 \nu_{1}}, s_{2 \nu_{2}}\right)+\frac{1}{k^{2}} z_{1}\left(s_{2 \nu_{1}}, s_{2 \nu_{2}}\right)+\frac{1}{k^{4}} z_{2}\left(s_{2 \nu_{1}}, s_{2 \nu_{2}}\right)+\ldots\right)
$$


where $s_{2 \nu_{i}}=2 k^{\nu_{i}-1} \xi_{2 \nu_{i}}$. The terms of this expansion are determined by the following partial differential scheme:

$f_{s_{2 \nu_{i}}}=c_{\nu_{i}} f^{\nu_{i}} f_{w}+\frac{1}{k^{2}} F_{1}^{\left(\nu_{i}\right)}\left(f, f_{w}, f_{w w}, f_{w w w}\right)+\cdots+\frac{1}{k^{2 g}} F_{g}^{\left(\nu_{i}\right)}\left(f, f_{w}, f_{w^{(2)}}, \ldots, f_{w^{(2 g+1)}}\right)+\left.\ldots\right|_{\text {evaluated at } w=1}$

where

$$
\begin{aligned}
f\left(s_{2 \nu_{1}}, s_{2 \nu_{2}} ; w\right) & =f_{0}\left(s_{2 \nu_{1}}, s_{2 \nu_{2}} ; w\right)+\frac{1}{k^{2}} f_{1}\left(s_{2 \nu_{1}}, s_{2 \nu_{2}} ; w\right)+\frac{1}{k^{4}} f_{2}\left(s_{2 \nu_{1}}, s_{2 \nu_{2}} ; w\right)+\ldots, \text { and } f_{g} \text { has the form } \\
f_{g}\left(s_{2 \nu_{1}}, s_{2 \nu_{2}} ; w\right) & =w^{1-2 g} z_{g}\left(w^{\nu_{1}-1} s_{2 \nu_{1}}, w^{\nu_{2}-1} s_{2 \nu_{2}}\right) .
\end{aligned}
$$

This partial differential scheme yields a hierarchy of partial differential equations in the same manner as in Theorem 2.2. The functionals $F_{g}^{\left(\nu_{i}\right)}$ are identical to the ones found in Theorem 2.2.

The $z_{g}\left(s_{2 \nu_{1}}, s_{2 \nu_{2}}\right)$ play the role of auxiliary functions in computing $e_{g}\left(-s_{2 \nu_{1}},-s_{2 \nu_{2}}\right)$. The differential equation determining $e_{g}\left(-s_{2 \nu_{1}},-s_{2 \nu_{2}}\right)$ remains largely unchanged from (5.19) because the construction in section 4.2 applies for the multi-time case as well:

Theorem 5.4. The $g$ 'th equation in the hierarchy of equations governing $e_{g}\left(t_{2 \nu_{1}}, t_{2 \nu_{2}}\right)$ is

$$
\begin{aligned}
& \frac{\partial^{2}}{\partial w^{2}}\left[w^{2-2 g} e_{g}\left(-w^{\nu_{1}-1} s_{2 \nu_{1}},-w^{\nu_{2}-1} s_{2 \nu_{2}}\right)\right]_{w=1} \\
& =-\sum_{n=1}^{g} \frac{2}{(2 n+2) !} \frac{\partial^{2 n+2}}{\partial w^{2 n+2}}\left[w^{2-2(g-n)} e_{g-n}\left(-w^{\nu_{1}-1} s_{2 \nu_{1}},-w^{\nu_{2}-1} s_{2 \nu_{2}}\right)\right] \\
& \quad+\text { the } k^{-2 g} \text { term of } \log \left[\sum_{n=0}^{\infty} \frac{1}{k^{2 n}} z_{n}\left(s_{2 \nu_{1}}, s_{2 \nu_{2}}\right)\right] .
\end{aligned}
$$

Equation (5.34) determines $e_{g}\left(-s_{2 \nu_{1}},-s_{2 \nu_{2}}\right)$ from a second order partial differential equation of $e_{g}$ with forcing terms depending on $e_{n}, n<g, z_{n}, n \leq g$, and their derivatives with respect to $s_{2 \nu_{1}}$ and $s_{2 \nu_{2}}$.

Theorem 5.5. The RHS of (5.34) will henceforth be denoted by drivers ${ }_{g}\left(s_{2 \nu_{1}}, s_{2 \nu_{2}}\right)$. The solution of (5.34) may be represented as

$$
\begin{aligned}
e_{g}\left(-s_{2 \nu_{1}},-s_{2 \nu_{2}}\right)= & \left(\nu_{1}-1\right)^{-1} \\
& {\left[s_{2 \nu_{1}}^{-(1-2 g) /\left(\nu_{1}-1\right)} \int_{0}^{s_{2 \nu_{1}}}\left(\hat{s}_{2 \nu_{1}}\right)^{\left(2-\nu_{1}-2 g\right) /\left(\nu_{1}-1\right)} \operatorname{drivers}_{g}\left(\hat{s}_{2 \nu_{1}}, u \hat{s}_{2 \nu_{1}}^{\left(\nu_{2}-1\right) /\left(\nu_{1}-1\right)}\right) d \hat{s}_{2 \nu_{2}}\right.} \\
& \left.-s_{2 \nu_{1}}^{-(2-2 g) /\left(\nu_{1}-1\right)} \int_{0}^{s_{2 \nu_{1}}}\left(\hat{s}_{2 \nu_{1}}\right)^{\left(3-\nu_{1}-2 g\right) /\left(\nu_{1}-1\right)} \operatorname{drivers}_{g}\left(\hat{s}_{2 \nu_{1}}, u \hat{s}_{2 \nu_{1}}^{\left(\nu_{2}-1\right) /\left(\nu_{1}-1\right)}\right) d \hat{s}_{2 \nu_{1}}\right] \\
& +K_{1}(u) s_{2 \nu_{1}}^{(2-2 g) /\left(\nu_{1}-1\right)}+K_{2}(u) s_{2 \nu_{1}}^{(1-2 g) /\left(\nu_{1}-1\right)},
\end{aligned}
$$

where $u=s_{2 \nu_{2}} s_{2 \nu_{1}}^{-\left(\nu_{2}-1\right) /\left(\nu_{1}-1\right)}$, and where $K_{1}(u)$ and $K_{2}(u)$ are analytic functions of $u$ in a neighborhood of $u=0$.

To prove Theorem [5.5 first we will show that $z_{0}\left(s_{2 \nu_{1}}, s_{2 \nu_{2}}\right)$ is given implicitly as the solution of an algebraic equation with coefficients depending on $s_{2 \nu_{1}}$ and $s_{2 \nu_{2}}$. Then we demonstrate that $z_{g}\left(s_{2 \nu_{1}}, s_{2 \nu_{2}}\right)$ are functions of $z_{0}\left(s_{2 \nu_{1}}, s_{2 \nu_{2}}\right)$ and $z_{0}\left(s_{2 \nu_{1}}, 0\right)$. Finally we compute $e_{g}\left(-s_{2 \nu_{1}},-s_{2 \nu_{2}}\right)$ by integrating the partial differential equation in Theorem 5.34

The first order terms in the hierarchy of Theorem [5.3 are the pair of equations

$$
\begin{aligned}
\frac{d z_{0}}{d s_{2 \nu_{1}}} & =c_{\nu_{1}} z_{0}^{\nu_{1}}\left(z_{0}+\left(\nu_{1}-1\right) s_{2 \nu_{1}} \frac{d z_{0}}{d s_{2 \nu_{1}}}+\left(\nu_{2}-1\right) s_{2 \nu_{2}} \frac{d z_{0}}{d s_{2 \nu_{2}}}\right) \\
\frac{d z_{0}}{d s_{2 \nu_{2}}} & =c_{\nu_{2}} z_{0}^{\nu_{2}}\left(z_{0}+\left(\nu_{1}-1\right) s_{2 \nu_{1}} \frac{d z_{0}}{d s_{2 \nu_{1}}}+\left(\nu_{2}-1\right) s_{2 \nu_{2}} \frac{d z_{0}}{d s_{2 \nu_{2}}}\right)
\end{aligned}
$$

or in vector notation

$$
\mathbf{M}\left(\begin{array}{l}
\frac{d z_{0}}{d s_{2 \nu_{1}}} \\
\frac{d z_{0}}{d s_{2 \nu_{2}}}
\end{array}\right)=\left(\begin{array}{l}
c_{\nu_{1}} z_{0}^{\nu_{1}+1} \\
c_{\nu_{2}} z_{0}^{\nu_{2}+1}
\end{array}\right)
$$


where

$$
\mathbf{M}=\left(\begin{array}{cc}
1-c_{\nu_{1}}\left(\nu_{1}-1\right) s_{2 \nu_{1}} z_{0}^{\nu_{1}} & -c_{\nu_{1}}\left(\nu_{2}-1\right) s_{2 \nu_{2}} z_{0}^{\nu_{1}} \\
-c_{\nu_{2}}\left(\nu_{1}-1\right) s_{2 \nu_{1}} z_{0}^{\nu_{2}} & 1-c_{\nu_{2}}\left(\nu_{2}-1\right) s_{2 \nu_{2}} z_{0}^{\nu_{2}}
\end{array}\right)
$$

Invert $\mathbf{M}$ in (5.36) to find a pair of ordinary differential equations for $z_{0}$ :

$$
\frac{d z_{0}}{d s_{2 \nu_{i}}}=-\frac{c_{\nu_{i}} z_{0}^{\nu_{i}+1}}{-1+c_{\nu_{1}}\left(\nu_{1}-1\right) s_{2 \nu_{1}} z_{0}^{\nu_{1}}+c_{\nu_{2}}\left(\nu_{2}-1\right) s_{2 \nu_{2}} z_{0}^{\nu_{2}}}
$$

which, with initial condition $z_{0}(0,0)=1$, is satisfied implicitly by the solution of

$$
1=z_{0}-c_{\nu_{1}} s_{2 \nu_{1}} z_{0}^{\nu_{1}}-c_{\nu_{2}} s_{2 \nu_{2}} z_{0}^{\nu_{2}}
$$

which is regular at $\left(s_{2 \nu_{1}}, s_{2 \nu_{2}}\right)=(0,0)$.

The $g^{\text {th }}$ pair of equations in the hierarchy of partial differential equations in Theorem 5.3 is

$$
\mathbf{M}\left(\begin{array}{c}
\frac{d z_{g}}{d s_{2 \nu_{1}}} \\
\frac{d z_{g}}{d s_{2 \nu_{2}}}
\end{array}\right)=z_{g} \mathbf{G}_{g}+\mathbf{F}_{g} ;
$$

where

$$
\begin{aligned}
\mathbf{G}_{g} & =\left(\begin{array}{l}
c_{\nu_{1}}\left(\nu_{1}+1-2 g\right) z_{0}^{\nu_{1}}+c_{\nu_{1}} \nu_{1}\left(\nu_{1}-1\right) s_{2 \nu_{1}} z_{0}^{\nu_{1}-1} \frac{d z_{0}}{d s_{2} \nu_{1}}+c_{\nu_{1}} \nu_{1}\left(\nu_{2}-1\right) s_{2 \nu_{2}} z_{0}^{\nu_{2}-1} \frac{d z_{0}}{d s_{2 \nu_{2}}} \\
c_{\nu_{2}}\left(\nu_{2}+1-2 g\right) z_{0}^{\nu_{2}}+c_{\nu_{2}} \nu_{2}\left(\nu_{1}-1\right) s_{2 \nu_{1}} z_{0}^{\nu_{2}-1} \frac{d z_{0}}{d s_{2 \nu_{1}}}+c_{\nu_{2}} \nu_{2}\left(\nu_{2}-1\right) s_{2 \nu_{2}} z_{0}^{\nu_{2}-1} \frac{d z_{0}}{d s_{2 \nu_{2}}}
\end{array}\right) \\
\mathbf{F}_{g} & =\left.\left(\begin{array}{l}
\operatorname{Forcing}_{g}^{\left(\nu_{1}\right)} \\
\operatorname{Forcing}_{g}^{\left(\nu_{2}\right)}
\end{array}\right)\right|_{w=1},
\end{aligned}
$$

and where $\operatorname{Forcing}_{g}^{\left(\nu_{i}\right)}$ is given by (2.8) with

$$
f_{g}\left(s_{2 \nu_{1}}, s_{2 \nu_{2}} ; w\right)=w^{1-2 g} z_{g}\left(w^{\nu_{1}-1} s_{2 \nu_{1}}, w^{\nu_{2}-1} s_{2 \nu_{2}}\right) \text {. }
$$

It is useful at this stage to make the following change of variables: let $z_{0}\left(s_{2 \nu_{1}}, 0\right)=y_{0}$ and denote $z_{0}\left(s_{2 \nu_{1}}, s_{2 \nu_{2}}\right)$ as $z_{0}$. The constraint equation (5.37) becomes:

$$
\begin{aligned}
& 1=y_{0}-c_{\nu_{1}} s_{2 \nu_{1}} y_{0}^{\nu_{1}} \\
& 1=z_{0}-c_{\nu_{1}} s_{2 \nu_{1}} z_{0}^{\nu_{1}}-c_{\nu_{2}} s_{2 \nu_{2}} z_{0}^{\nu_{2}},
\end{aligned}
$$

and one can solve equations (5.39) and (5.40) for $\left(s_{2 \nu_{1}}, s_{2 \nu_{2}}\right)$ as functions of $\left(y_{0}, z_{0}\right)$,

$$
\begin{aligned}
& s_{2 \nu_{1}}=\left(y_{0}-1\right)\left(c_{\nu_{1}} y_{0}^{\nu_{1}}\right)^{-1} \\
& s_{2 \nu_{2}}=\left(y_{0}^{\nu_{1}}\left(z_{0}-1\right)-z_{0}^{\nu_{1}}\left(y_{0}-1\right)\right)\left(c_{\nu_{2}} y_{0}^{\nu_{1}} z_{0}^{\nu_{2}}\right)^{-1},
\end{aligned}
$$

and then differentiate equation (5.39 and 5.40) with respect to $s_{2 \nu_{1}}$ and $s_{2 \nu_{2}}$ and solve for $\frac{d y_{0}}{d s_{2 \nu_{1}}}$, $\frac{d z_{0}}{d s_{2 \nu_{1}}}$ and $\frac{d z_{0}}{d s_{2 \nu_{2}}}$ as functions of $\left(y_{0}, z_{0}\right)$ :

$$
\begin{aligned}
\frac{d y_{0}}{d s_{2 \nu_{1}}} & =c_{\nu_{1}} y_{0}^{\nu_{1}+1}\left(\nu_{1}-\left(\nu_{1}-1\right) y_{0}\right)^{-1} \\
\frac{d y_{0}}{d s_{2 \nu_{2}}} & =0 \\
\frac{d z_{0}}{d s_{2 \nu_{1}}} & =c_{\nu_{1}} z_{0}^{\nu_{1}}\left(1-\nu_{1} c_{\nu_{1}} s_{2 \nu_{1}} z_{0}^{\nu_{1}-1}-\nu_{2} c_{\nu_{2}} s_{2 \nu_{2}} z_{0}^{\nu_{2}-1}\right)^{-1} \\
\frac{d z_{0}}{d s_{2 \nu_{2}}} & =c_{\nu_{2}} z_{0}^{\nu_{2}}\left(1-\nu_{1} c_{\nu_{1}} s_{2 \nu_{1}} z_{0}^{\nu_{1}-1}-\nu_{2} c_{\nu_{2}} s_{2 \nu_{2}} z_{0}^{\nu_{2}-1}\right)^{-1} .
\end{aligned}
$$

Changing variables in the system of differential equations (5.38) to differential equations for $z_{g}$ as a function of $\left(y_{0}, z_{0}\right)$; where $y_{0}$ evolves from 1 to $z_{0}\left(s_{2 \nu_{1}}, 0\right)$ and $z_{0}$ from $z_{0}\left(s_{2 \nu_{1}}, 0\right)$ to $z_{0}\left(s_{2 \nu_{1}}, s_{2 \nu_{2}}\right)$, equation (5.38) becomes

$$
\mathbf{M C}\left(\begin{array}{l}
\frac{d z_{g}}{d y_{0}} \\
\frac{d z_{g}}{d z_{0}}
\end{array}\right)=z_{g} \mathbf{G}_{g}+\mathbf{F}_{g}
$$


where

$$
\mathbf{C}=\left(\begin{array}{cc}
c_{\nu_{1}} y_{0}^{\nu_{1}+1}\left(\nu_{1}-\left(\nu_{1}-1\right) y_{0}\right)^{-1} & c_{\nu_{1}} z_{0}^{\nu_{1}}\left(1-\nu_{1} c_{\nu_{1}} s_{2 \nu_{1}} z_{0}^{\nu_{1}-1}-\nu_{2} c_{\nu_{2}} s_{2 \nu_{2}} z_{0}^{\nu_{2}-1}\right)^{-1} \\
0 & c_{\nu_{2}} z_{0}^{\nu_{2}}\left(1-\nu_{1} c_{\nu_{1}} s_{2 \nu_{1}} z_{0}^{\nu_{1}-1}-\nu_{2} c_{\nu_{2}} s_{2 \nu_{2}} z_{0}^{\nu_{2}-1}\right)^{-1}
\end{array}\right)
$$

and $s_{2 \nu_{1}}$ and $s_{2 \nu_{2}}$ are given by equations (5.41) and (5.42).

If $\mathbf{M C}$ is invertible then $z_{g}$ can be found by integrating the differential equation:

$$
\frac{d z_{g}}{d z_{0}}=z_{g}\left[\mathbf{C}^{-1} \mathbf{M}^{-1} \mathbf{G}_{g}\right]_{2}+\left[\mathbf{C}^{-1} \mathbf{M}^{-1} \mathbf{F}_{g}\right]_{2},
$$

where $[\mathbf{V}]_{2}$ denotes the second component of the vector $\mathbf{V}$. The initial condition is that $z_{g}\left(y_{0}, z_{0}=y_{0}\right)$ agrees with Theorem 2.3 with $\nu=\nu_{1}$.

We will now prove Theorem [5.5] Start by expanding the LHS of (5.34):

$$
\begin{aligned}
& (2-2 g)(1-2 g) e_{g}-\left(\nu_{1}-1\right)\left(\nu_{1}+2-4 g\right) s_{2 \nu_{1}} e_{g s_{2 \nu_{1}}}-\left(\nu_{2}-1\right)\left(\nu_{2}+2-4 g\right) s_{2 \nu_{2}} e_{g s_{2 \nu_{2}}} \\
& +\left(\nu_{1}-1\right)^{2} s_{2 \nu_{1}}^{2} e_{g s_{2 \nu_{1}} s_{2 \nu_{1}}}+2\left(\nu_{1}-1\right)\left(\nu_{2}-1\right) s_{2 \nu_{1}} s_{2 \nu_{2}} e_{g s_{2 \nu_{1}} s_{2 \nu_{2}}}+\left(\nu_{2}-1\right)^{2} s_{2 \nu_{2}}^{2} e_{g s_{2 \nu_{2}} s_{2 \nu_{2}}}=\operatorname{drivers} g\left(s_{2 \nu_{1}}, s_{2 \nu_{2}}\right) .
\end{aligned}
$$

Changing variables to

$$
\left(\hat{s}_{2 \nu_{1}}, u\right)=\left(s_{2 \nu_{1}}, s_{2 \nu_{2}} s_{2 \nu_{1}}^{-\left(\nu_{2}-1\right) /\left(\nu_{1}-1\right)}\right)
$$

induces

$$
\left(\begin{array}{c}
\hat{s}_{2 \nu_{1}} \frac{\partial}{\partial \hat{s}_{2 \nu_{1}}} \\
u \frac{\partial}{\partial u}
\end{array}\right)=\left(\begin{array}{c}
s_{2 \nu_{1}} \frac{\partial}{\partial s_{2 \nu_{1}}}+\frac{\left(\nu_{2}-1\right)}{\left(\nu_{1}-1\right)} s_{2 \nu_{2}} \frac{\partial}{\partial s_{2 \nu_{2}}} \\
s_{2 \nu_{2}} \frac{\partial}{\partial s_{2 \nu_{2}}}
\end{array}\right) .
$$

Equation (5.45) may be rewritten in these new variables as:

$$
\left(\nu_{1}-1\right)^{2}\left(\hat{s}_{2 \nu_{1}} \frac{\partial}{\partial \hat{s}_{2 \nu_{1}}}+\frac{(1-2 g)}{\left(\nu_{1}-1\right)}\right)\left(\hat{s}_{2 \nu_{1}} \frac{\partial}{\partial \hat{s}_{2 \nu_{1}}}+\frac{(2-2 g)}{\left(\nu_{1}-1\right)}\right) e_{g}=\operatorname{drivers}_{g}\left(\hat{s}_{2 \nu_{1}}, u \hat{s}_{2 \nu_{1}}^{\left(\nu_{2}-1\right) /\left(\nu_{1}-1\right)}\right),
$$

whose LHS is identical to the differential equation (5.20) for $e_{g}\left(-s_{2 \nu_{1}}\right)$.

Equation (5.46) can be integrated to give

$$
\begin{aligned}
e_{g}\left(-s_{2 \nu_{1}},-s_{2 \nu_{2}}\right)= & \left(\nu_{1}-1\right)^{-2} s_{2 \nu_{1}}^{-(2-2 g) /\left(\nu_{1}-1\right)} \int_{0}^{s_{2 \nu_{1}}}\left(s_{2 \nu_{1}}^{\prime}\right)^{\left(2-\nu_{1}\right) /\left(\nu_{1}-1\right)} \int_{0}^{s_{2 \nu_{1}}^{\prime}}\left(s_{2 \nu_{1}}^{\prime \prime}\right)^{\left(2-\nu_{1}-2 g\right) /\left(\nu_{1}-1\right)} \\
& \cdot \operatorname{drivers}\left(s_{2 \nu_{1}}^{\prime \prime}, u s_{2 \nu_{1}}^{\prime \prime\left(\nu_{2}-1\right) /\left(\nu_{1}-1\right)}\right) d s_{2 \nu_{1}}^{\prime \prime} d s_{2 \nu_{1}}^{\prime} \\
& +K_{1}(u) s_{2 \nu_{1}}^{(2-2 g) /\left(\nu_{1}-1\right)}+K_{2}(u) s_{2 \nu_{1}}^{(1-2 g) /\left(\nu_{1}-1\right)},
\end{aligned}
$$

where $u=s_{2 \nu_{2}} s_{2 \nu_{1}}^{-\left(\nu_{2}-1\right) /\left(\nu_{1}-1\right)}$ and $K_{1}(u)$ and $K_{2}(u)$ are functions of $u$ only.

Switch the order of integration in (5.47),

$$
\begin{aligned}
e_{g}\left(-s_{2 \nu_{1}},-s_{2 \nu_{2}}\right)= & \left(\nu_{1}-1\right)^{-2} s_{2 \nu_{1}}^{-(2-2 g) /\left(\nu_{1}-1\right)} \int_{0}^{s_{2 \nu_{1}}} \int_{s_{2 \nu_{1}}^{\prime \prime}}^{s_{2 \nu_{1}}}\left(s_{2 \nu_{1}}^{\prime}\right)^{\left(2-\nu_{1}\right) /\left(\nu_{1}-1\right)}\left(s_{2 \nu_{1}}^{\prime \prime}\right)^{\left(2-\nu_{1}-2 g\right) /\left(\nu_{1}-1\right)} \\
& \cdot \operatorname{drivers}_{g}\left(s_{2 \nu_{1}}^{\prime \prime}, u s^{\prime \prime}{ }_{2 \nu_{1}}^{\left(\nu_{2}-1\right) /\left(\nu_{1}-1\right)}\right) d s_{2 \nu_{1}}^{\prime} d s_{2 \nu_{1}}^{\prime \prime} \\
& +K_{1}(u) s_{2 \nu_{1}}^{(2-2 g) /\left(\nu_{1}-1\right)}+K_{2}(u) s_{2 \nu_{1}}^{(1-2 g) /\left(\nu_{1}-1\right)}
\end{aligned}
$$

and carry out the $s_{2 \nu_{1}}^{\prime}$ integral in (5.48) to find

$$
\begin{aligned}
& e_{g}\left(-s_{2 \nu_{1}},-s_{2 \nu_{2}}\right)=\left(\nu_{1}-1\right)^{-1} \\
& {\left[s_{2 \nu_{1}}^{-(1-2 g) /\left(\nu_{1}-1\right)} \int_{0}^{s_{2 \nu_{1}}}\left(s_{2 \nu_{1}}^{\prime \prime}\right)^{\left(2-\nu_{1}-2 g\right) /\left(\nu_{1}-1\right)} \operatorname{drivers}_{g}\left(s_{2 \nu_{1}}^{\prime \prime}, u s^{\prime \prime}{ }_{2 \nu_{1}}^{\left(\nu_{2}-1\right) /\left(\nu_{1}-1\right)}\right) d s_{2 \nu_{2}}^{\prime \prime}\right.} \\
& \left.-s_{2 \nu_{1}}^{-(2-2 g) /\left(\nu_{1}-1\right)} \int_{0}^{s_{2 \nu_{1}}}\left(s_{2 \nu_{1}}^{\prime \prime}\right)^{\left(3-\nu_{1}-2 g\right) /\left(\nu_{1}-1\right)} \operatorname{drivers}{ }_{g}\left(s_{2 \nu_{1}}^{\prime \prime}, u s^{\prime \prime}{ }_{2 \nu_{1}}^{\left(\nu_{2}-1\right) /\left(\nu_{1}-1\right)}\right) d s_{2 \nu_{1}}^{\prime \prime}\right] \\
& +K_{1}(u) s_{2 \nu_{1}}^{(2-2 g) /\left(\nu_{1}-1\right)}+K_{2}(u) s_{2 \nu_{1}}^{(1-2 g) /\left(\nu_{1}-1\right)} .
\end{aligned}
$$

This proves formula (5.35) of Theorem 5.5 


\section{Conclusions}

In this paper we have made a detailed study of the coefficients $e_{g}(x, t)$ in the asymptotic expansion of the logarithm of the random matrix partition function (1.7) for a single non-trivial time parameter, $t=t_{2 \nu}$ and $x=k / N$ near 1 . These analytic coefficients are generating functions for the enumeration of $g$-maps. In particular,

(1) We derived a hierarchy of differential equations for these generating functions.

(2) We described a procedure for solving these differential equations.

(3) Along the way to deriving the hierarchy we also derive an hierarchy of forced Burgers type equations for the auxiliary coefficients which we denote $z_{g}(s)$; these are of combinatorial interest in their own right in that

$$
\frac{\partial^{n}}{\partial s^{n}} z_{g}(0)=\sharp\{\text { two-legged } g \text {-maps with } n 2 \nu \text {-valent vertices }\} \text {. }
$$

A leg is an edge emerging from a univalent vertex; so that the leg is the only edge incident to that vertex.

(4) We have calculated explicit formulae for $e_{g}(-s)$ for small values of $g$. The $s$-derivatives, of sufficiently large order, turn out to be rational functions of the endpoints squared, $\beta^{2}=4 z_{0}$. The endpoints referred to here are the endpoints of the support of the associated equilibrium measure. Moreover, the coefficients appearing in these expressions are rational constants.

The random matrix partition functions and their relations to graphical enumeration through diagrammatic expansion offer powerful tools for bringing methods of complex analysis to bear on fundamental questions in diverse fields such as statistical mechanics [13] and combinatorics 26, 5]. However, real progress in this regard has been hampered by the fact that, up till now, these connections have been based on formal procedures and conjectures.

The results in this paper place many of these connections, for the large $N$ expansion of the UE partition function, on a rigorous foundation. Moreover, the methods presented here, based on continuum limits of the Toda Lattice hierarchy, have yielded novel and effective procedures for explicitly calculating the relevant asymptotic generating functions. We hope that these results will help to spur renewed application of complex analytic methods in problems of statistical physics and combinatorics. Some results in this direction will appear in future work 22,23$]$.

These analytical tools also point the way to novel combinatorial reults. The calculations mentioned in the last item of the list above, strongly motivate the

Conjecture: The $s$-derivatives of sufficiently high order of the generating functions, $e_{g}$, for fixed genus with arbitrary vertex valence number, can be expressed as rational functions of the endpoints of support of the equilibrium measure. Moreover, the coefficients appearing in these expressions are rational constants.

As far as we know, such a conjecture has not appeared in the literature on combinatorics of maps.

Another manuscript in preparation [16] rigorously establishes a nonlocal representation for the $e_{g}(1, t)$. In the physics literature such representations are referred to as loop equations [2]. We expect this to provide elegant methods that, together with the present manuscript, could enable us to prove the previous conjecture. Moreover, the generalizations of this loop equation together with the results of section 5.11 can help to guide the characterization of $e_{g}(\mathbf{t})$ as a function of multiple times. The derivation of explicit closed form expressions for $e_{g}(\mathbf{t})$ will have relevance to a number of other current research programs in the statistical mechanics of combinatorial analysis such as random graphs, random tilings and polynuclear growth models.

Acknowledgements: V. U. Pierce would like to thank The University of Arizona, Brandeis University, Midwestern State University, and The Ohio State University for their hospitality and support; and Mark Adler and Yuji Kodama for many helpful discussions and their encouragement. 


\section{Appendix A.}

In this appendix we show how the expansion (3.25) for the integral (3.24) was derived. Define

$$
d_{j}=\int_{\beta}^{\lambda} s^{2 j} \sqrt{s^{2}-\beta^{2}} d s
$$

then (3.24) is

$$
\int_{\beta}^{\lambda} h(s) \sqrt{s^{2}-\beta^{2}} d s=\frac{1}{x}\left(d_{0}+\sum_{j=0}^{\nu-1} h_{j} d_{j}\right)
$$

Integration by parts $\left(u=s^{2 j-1}\right.$ and $\left.d v=s \sqrt{s^{2}-\beta^{2}}\right)$ of $d_{j}$ gives the equation

$$
d_{j}=\frac{1}{3} \lambda^{2 j-1}\left(\lambda^{2}-\beta^{2}\right) \sqrt{\lambda^{2}-\beta^{2}}-\int \frac{(2 j-1)}{3} s^{2 j-2}\left(s^{2}-\beta^{2}\right) \sqrt{s^{2}-\beta^{2}} d s .
$$

Equation A.2 produces a recursion relation for $d_{j}$ :

$$
d_{j}=\frac{1}{2} \frac{1}{j+1} \lambda^{2 j-1}\left(\lambda^{2}-\beta^{2}\right) \sqrt{\lambda^{2}-\beta^{2}}+\frac{1}{2} \frac{(2 j-1)}{j+1} \beta^{2} d_{j-1} .
$$

The initial condition of this recursion is

$$
d_{0}=\int_{\beta}^{\lambda} \sqrt{\lambda^{2}-\beta^{2}} d \lambda=\frac{1}{2} \lambda \sqrt{\lambda^{2}-\beta^{2}}-\frac{1}{2} \beta^{2} \log \left|\frac{\lambda}{\beta}+\sqrt{\lambda^{2}-\beta^{2}}\right| .
$$

The recursion relation A.3 , A.4 is solved by

$$
\begin{aligned}
d_{j}= & S_{j}^{(1)}(\lambda)\left(\lambda^{2}-\beta^{2}\right) \frac{\sqrt{\lambda^{2}-\beta^{2}}}{\lambda}+S_{j}^{(2)}(\lambda) \frac{\sqrt{\lambda^{2}-\beta^{2}}}{\lambda} \\
& +S_{j}^{(3)}(\lambda) \log \left(\frac{\lambda}{\beta}+\frac{\sqrt{\lambda^{2}-\beta^{2}}}{\beta}\right), j \in \mathbb{N} .
\end{aligned}
$$

The functions $S_{j}^{(2)}$ and $S_{j}^{(3)}$ are simple. We find the expressions:

$$
\begin{aligned}
S_{j}^{(2)} & =\frac{\beta^{2 j}}{4^{j}}\left(\begin{array}{c}
2 j-1 \\
j-1
\end{array}\right) \frac{1}{j+1} \lambda^{2}=\frac{v_{j}}{\beta^{2}} \lambda^{2}, \\
S_{j}^{(3)} & =-\frac{\beta^{2 j}}{4^{j}}\left(\begin{array}{c}
2 j-1 \\
j-1
\end{array}\right) \frac{1}{j+1}=-v_{j} .
\end{aligned}
$$

The case of $S_{j}^{(1)}$ is more complicated; after some work we have

$$
S_{j}^{(1)}=\frac{1}{2^{j}}\left(\begin{array}{c}
2 j-1 \\
j-1
\end{array}\right) \frac{1}{j+1} \sum_{i=1}^{j} \frac{2^{j-2 i+1}(j-i+2) \beta^{2 i-2}}{\left(\begin{array}{c}
2 j-2 i+1 \\
j-i \\
39
\end{array}\right)} \lambda^{2 j-2 i+2}=\frac{v_{j}}{2} \sum_{i=1}^{j} \frac{\lambda^{2 i}}{v_{i}(i+1)} .
$$


The integral (3.24), in terms of $S_{j}^{(1)}, S_{j}^{(2)}$, and $S_{j}^{(3)}$ is

$$
\begin{aligned}
\int_{\beta}^{\lambda} h(s) \sqrt{s^{2}-\beta^{2}} d s= & \frac{1}{x}\left(d_{0}+\sum_{j=0}^{\nu-1} h_{j} d_{j}\right) \\
= & \frac{1}{x}\left(\sum_{j=0}^{\nu-1} h_{j} S_{j}^{(1)}\right)\left(\lambda^{2}-\beta^{2}\right) \frac{\sqrt{\lambda^{2}-\beta^{2}}}{\lambda} \\
& +\frac{1}{x}\left(S_{0}^{(2)}+\sum_{j=0}^{\nu-1} h_{j} S_{j}^{(2)}\right) \frac{\sqrt{\lambda^{2}-\beta^{2}}}{\lambda}+ \\
& \frac{1}{x}\left(S_{0}^{(3)}+\sum_{j=0}^{\nu-1} h_{j} S_{j}^{(3)}\right) \log \left(\frac{\lambda}{\beta}+\frac{\sqrt{\lambda^{2}-\beta^{2}}}{\beta}\right) .
\end{aligned}
$$

Using the relation (3.14) and the explicit expressions for the $S_{j}^{(k)}$ derived above, we finally arrive at the form of the expansion for (3.24) given in (3.25).

\section{REFERENCES}

1. M. Abramowitz and I. Stegun. Handbook of Mathematical Functions, U.S. Govt. Printing Office, Washington, D.C., 1972.

2. J. Ambjorn, L. Chekhov, C.F. Kristjansen, Yu. Makeenko, Matrix Model Calculations Beyond the Spherical Limit Nuclear Physics B 404 (1993), 127-172.

3. S. Albeverio, L. Pastur, and M. Shcherbina. On the $1 / n$ expansion for some unitary invariant ensembles of random matrices. Comm. Math. Phys. 224 (2001), 271-305.

4. M. Bauer and C. Itzykson. Triangulations, Discr. Math. 156 (1996) 29-81.

5. D. Bessis, X. Itzykson, and J.B. Zuber. Quantum Field Theory Techniques in Graphical Enumeration. Adv. Appl. Math. 1 (1980) 109-157.

6. A. Bloch, F. Golse, A. Uribe. Dispersionless Toda and Toeplitz operators. Duke Math. J. 117 (2003), 157-196.

7. J. Bouttier, P. DiFrancesco and E. Guitter. Census of Planar Maps: ¿From the One-Matrix Model Solution to a Combinatorial Proof. arXiv:cond-mat/0207682

8. P. Bleher and A. Its. Asymptotics of the partition function of a random matrix model. Ann. Inst. Fourier (Grenoble) 55 (2005), no. 6, 1943-2000.

9. M. Bousquet-Melou and G. Schaeffer. The Degree Distribution in Bipartite Planar Maps: Applications to the Ising Model. arXiv:math.CO/0211070

10. P. Deift, T. Kriecherbauer and K. T-R McLaughlin. New results on the equilibrium measure for logarithmic potentials in the presence of an external field. J. Approx. Thry., 95 (1998), 388-475.

11. P. Deift, T. Kriecherbauer, K.T.-R. McLaughlin, S. Venakides, and X. Zhou. Uniform asymptotics for polynomials orthogonal with respect to varying exponential weights and applications to universality questions in random matrix theory. Commun. Pure Appl. Math. 52 (11) (1999) 1335-1425.

12. P. Deift and K. T-R McLaughlin. A Continuum Limit of the Toda Lattice. Memoirs of the AMS, 131, no. 624, January 1998.

13. P. Di Francesco, P. Ginsparg and J. Zinn-Justin. 2D gravity and random matrices. Physics Reports 254 (1995) $1-133$.

14. N.M. Ercolani, H. Flaschka and S. Singer. The Geometry of the Full Toda Lattice. Progress in Mathematics 115 (1993) 181-226.

15. N.M. Ercolani and K. D. T-R McLaughlin. Asymptotics of the partition function for random matrices via Riemann-Hilbert techniques, and applications to graphical enumeration. Internat. Math. Research Notices 14, 755-820 (2003).

16. N.M. Ercolani and K. D. T-R McLaughlin. Loop Equations for the Large N Limit of Random Matrices. In Preparation.

17. A. Erdélyi, W. Magnus, F. Oberhettinger, and F. G. Tricomi. Higher Transcendental Functions, Vol. 1. New York: Krieger, 30-31, 1981.

18. H. Flaschka. Integrable Systems and Torus Actions, in Lectures on Integrable Systems, eds. O. Babelon, P. Cartier and Y. Kosmann-Schwarzbach. World Scientific, London, 43-101, 1994.

19. K. Johansson. On fluctuations of eigenvalues of random hermitian matrices. Duke Mathematics Journal 91 (1998), no. 1, 151-204.

20. M.L. Mehta. Random Matrices, 2nd Edition, Academic Press, San Diego, CA, 1991.

21. V. Pierce. The asymtptoic expansion of the partition function of random matrices. PhD Thesis, University of Arizona, 2004.

22. V. Pierce. Combinatoric Results to Planar Maps. In Preparation.

23. V. Pierce. An Algorithm for Map Enumeration. In Preparation.

24. E. B. Saff and V. Totik. Logarithmic Potentials with External Fields. New York: Springer-Verlag, 1997.

25. G. Szegő, Orthogonal Polynomials, AMS Colloquium Publications, Vol. 23, 1939. 
26. E. Witten. Two-Dimensional Gravity and Intersection Theory on Moduli Space. Surveys in Differential Geometry, vol 1, (1991), 243-310.

27. A. Zvonkin. How to Draw a Group. Discrete Math., vol 180, (1998) 403-413.

Dept. of Math, Univ. of Arizona, 520-621-2713, FAX: 520-626-5186

E-mail address: ercolani@math.arizona.edu

Dept. of Math., Univ. of Arizona

E-mail address: mcl@math.arizona.edu

Dept. of Math., The Ohio State University

E-mail address: vpierce@math.ohio-state.edu 